Check for updates

Cite this: Mater. Adv., 2020, 1,1575

Received 9th May 2020

Accepted 6th July 2020

DOI: 10.1039/d0ma00291g

rsc.li/materials-advances

\section{What triggers dye adsorption by metal organic frameworks? The current perspectives}

\author{
M. Shahnawaz Khan, (D) Mohd Khalid (D) * and M. Shahid (D) *
}

\begin{abstract}
Coordination chemistry has always been the most important tool to envisage the material importance of metal-organic systems. The chemistry involved plays a key role in determining structural features, which are responsible for tuning the properties of materials. In the present review, the current trends of metal organic frameworks (MOFs), their structural properties and dye adsorption related to their structural architecture together with other parameters affecting adsorption phenomena are discussed. The content of hazardous dyes such as methylene blue, rhodamine B, and methyl orange has been continuously increasing in wastewater, and therefore, considering environmental safety, their removal is urgent. The present survey demonstrates the important role of MOFs in dye adsorption and separation. Their structure activity relationship, which is the most important factor, is elaborated through the current scenario. The mechanisms involved in the adsorption are also illustrated and explained. The mechanisms support that, besides the porous nature of MOFs, various non-covalent interactions also play a significant role in enhancing the adsorption of dyes. This review presents the modern trends of dye adsorption by MOFs and opens new doors for the further tuning of their structural features to modulate the adsorption/separation of hazardous materials, which will definitely guide the future endeavors of coordination chemists.
\end{abstract}

Functional Inorganic Materials Lab (FIML), Department of Chemistry,

Aligarh Muslim University, Aligarh 202002, India.E-mail:khalid215@gmail.com, shahid81chem@gmail.com

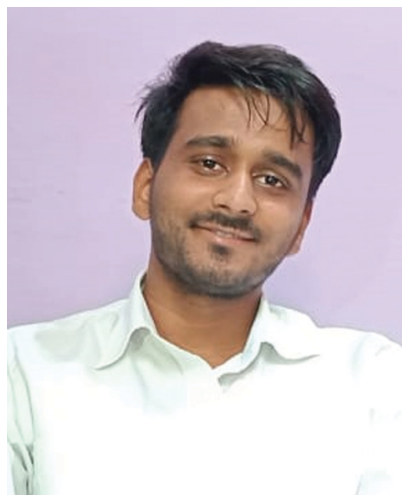

M. Shahnawaz Khan reputed journals. Currently, he is working on the project "Inorganic functional material as an adsorbent for hazardous substances in an aqueous medium." He works on designing metal organic frameworks and their functional applications in dye adsorption and degradation. He is a team member of the Functional Inorganic Materials Lab (FIML).

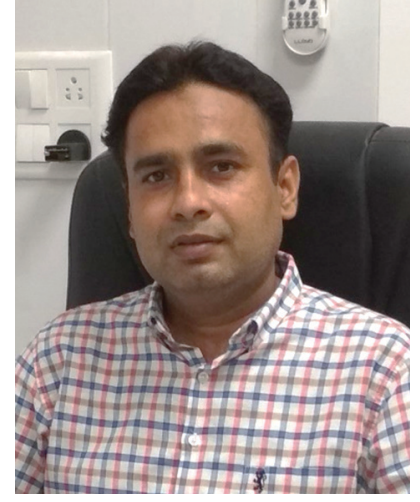

Mohd Khalid
Dr Mohd Khalid was born in Jaunpur, Uttar Pradesh India. He completed his BSc, MSc and $\mathrm{PhD}$ degrees in Chemistry from Aligarh Muslim University, Aligarh, India. He is an Assistant Professor in Department of Chemistry, AMU, Aligarh. He has been teaching Inorganic Chemistry to undergraduate and postgraduate students since last 10 years. He has published more than forty papers in international journals of repute. He is actively involved in design and X-ray crystallographic investigations of novel polynuclear clusters and metal organic frameworks (MOFs), and their material applications in sensing and dye adsorption. He has also got the UGC Start-Up Grant to work on such molecules of functional importance. He has also participated in various national and international conferences and symposia to present his work. He is a part of the Functional Inorganic Materials Lab (FIML). 


\section{Introduction}

Currently, the treatment of wastewater is challenging due to increasing amount of pollutant species from various sources. ${ }^{1}$ Water pollution has expanded in the last few decades, and has become the most challenging subject globally. It is predicted that by the end of 2025, people residing in desert regions will face a water shortage. ${ }^{1,2}$ Although gathering freshwater or groundwater from saltwater is an easy way to overcome this serious issue, this is impossible for communities with scarce resources $^{2}$ and those living in remote territories. The concern of water pollution is still ongoing, where each year a high volume of wastewater is released into fresh and running water in the aquatic environment. ${ }^{3}$ Thus, water purification, water reuse, and drinkable water have attracted much attention (Fig. 1). However, they face several hurdles, such as potential chronic toxicity, operational cost, and public recognition. ${ }^{4,5}$ Scientifically, the establishment of sharing systems with centralized pieces of equipment has to occur, and diverse technologies can be installed depending on the restricted conditions and requirements. Thus, the ultimate management of water and water quality must be maintained at a good standard. ${ }^{6}$ Separate from all the other types of wastewater, dye wastewater has attracted considerable recognition. In the last century, the discharge of dyes into the atmosphere from the printing and dyeing industries has progressed significantly. ${ }^{7}$ Organic dyes are everyday compounds and are used enormously in numerous fields for coloring textiles, paper, rubber, leather, printing, plastic, etc. ${ }^{8}$ Mainly, the textile industry utilizes more than 700,000 tons of dye, and is one of three principal pollutants

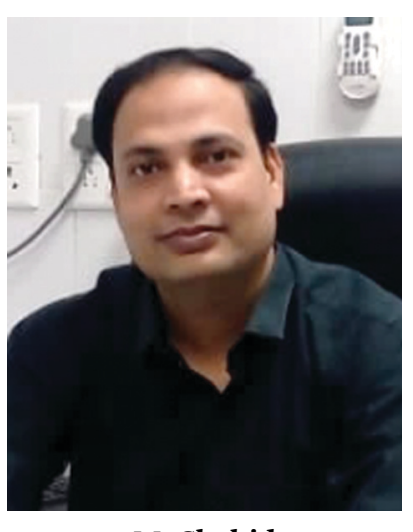

Dr Shahid is an Assistant Professor at Department of Chemistry, Aligarh Muslim University, Aligarh, India. He has been teaching Inorganic Chemistry to the under and post graduate students since 2010. He received his BS and MS degrees from CSJM University, Kanpur. He holds a $\mathrm{PhD}$ degree in Inorganic Chemistry from Aligarh Muslim University, Aligarh. After completing PhD, he worked as a Fast Track Young Scientist in the same department. He received many fellowships and awards given by various agencies of Govt. of India. He is a part of the Functional Inorganic Materials Lab (FIML). His research group is actively involved in development of cages, clusters and MOFs to serve as magnets, coolers, sensors and adsorbent materials. He has successfully completed many research projects funded by SERB (DST), UGC and ICMR and is currently involved in some major projects on developing inorganic functional materials. He has more than 80 research papers to his credit and filed one Indian Patent in July 2020. in modern times. Most of the utilized dyes are organic compounds, which are teratogenic and carcinogenic. ${ }^{9-12}$ The traditional approaches used for the remediation of wastewater include sedimentation, coagulation, and chemical and membrane technologies. However, although they are most extensively used, these methods do not eliminate contaminants effectively from wastewater and they produce secondary pollutants (Fig. 2). ${ }^{13}$ Industrial wastewater comprises a full spectrum of organic pollutants, oil, detergents, and other carcinogenic organic compounds, ${ }^{14}$ which have different harmful effects on humans and animals. ${ }^{15}$ These carcinogenic pollutants in wastewater cannot be fully degraded using the abovementioned conservative methods, which can be lethal and harmful to biological processes. Thus, two approaches have attracted much interest in treating organic pollutants in wastewater as follows: (1) The use of an appropriate adsorbent for the adsorption of organic pollutants from wastewater using polymeric resins, biomaterials, activated carbon, clay minerals, zeolites and industrial solid waste (Fig. 3). ${ }^{16-18}$ The adsorption process is very simple and cost effective. ${ }^{19}$ In this field, activated carbon is the most widely accepted adsorbent because of its efficacy in the adsorption process. ${ }^{20}$ However, its application is restricted due to its high cost and very difficult use. ${ }^{21}$ Accordingly, many new methods have been explored to replace the conventional activated carbon for the adsorption process. (2) The second approach involves removing organic pollutants via degradation through advanced oxidation processes. The degradation process involves the generation of highly reactive species, which oxidize toxic organic compounds into simple compounds with lower toxicity or fully degrade them to form $\mathrm{CO}_{2}$ and water.

One of the most common organic pollutants is aromatic organic dyes in the wastewater from industries. Since becoming aware of the hazardous nature of dyeing materials, scientists have worked hard to defeat this obstacle, and accordingly, have developed numerous chemical, biological and physical methods for the treatment of wastewater. These techniques include flocculation, adsorption, photocatalysis, biodegradation, coagulation, electrochemical process, membrane separation, advanced oxidation processes and ion-exchange. The main advantages and disadvantages of these techniques are shown in Table 1. The utilization of biological methods is comprehensive due to its eco-friendly and simple procedures compared to other methods. In the biological process, many microorganisms are used for the decolourization of dyes under varying conditions. However, there are various disadvantage associated with the biological process, which requires a strict external environment and suitable conditions such as temperature and $\mathrm{pH}$. The principle limitation of the biological method is that it requires a bioreactor to be established, which demands a specific amount of land, makes the process more intricate, and requires much more time. ${ }^{22}$ Furthermore, the biological method does not have exceptional efficiency in degrading dyes due to the stability and rigid structure of these synthetic dyes. The main concern with biological processes is that there will be a lot of sludge to be disposed. On the other hand, the chemical process is beneficial and practical, but the deposition of mud adds increase the cost of the process. The main downside of 


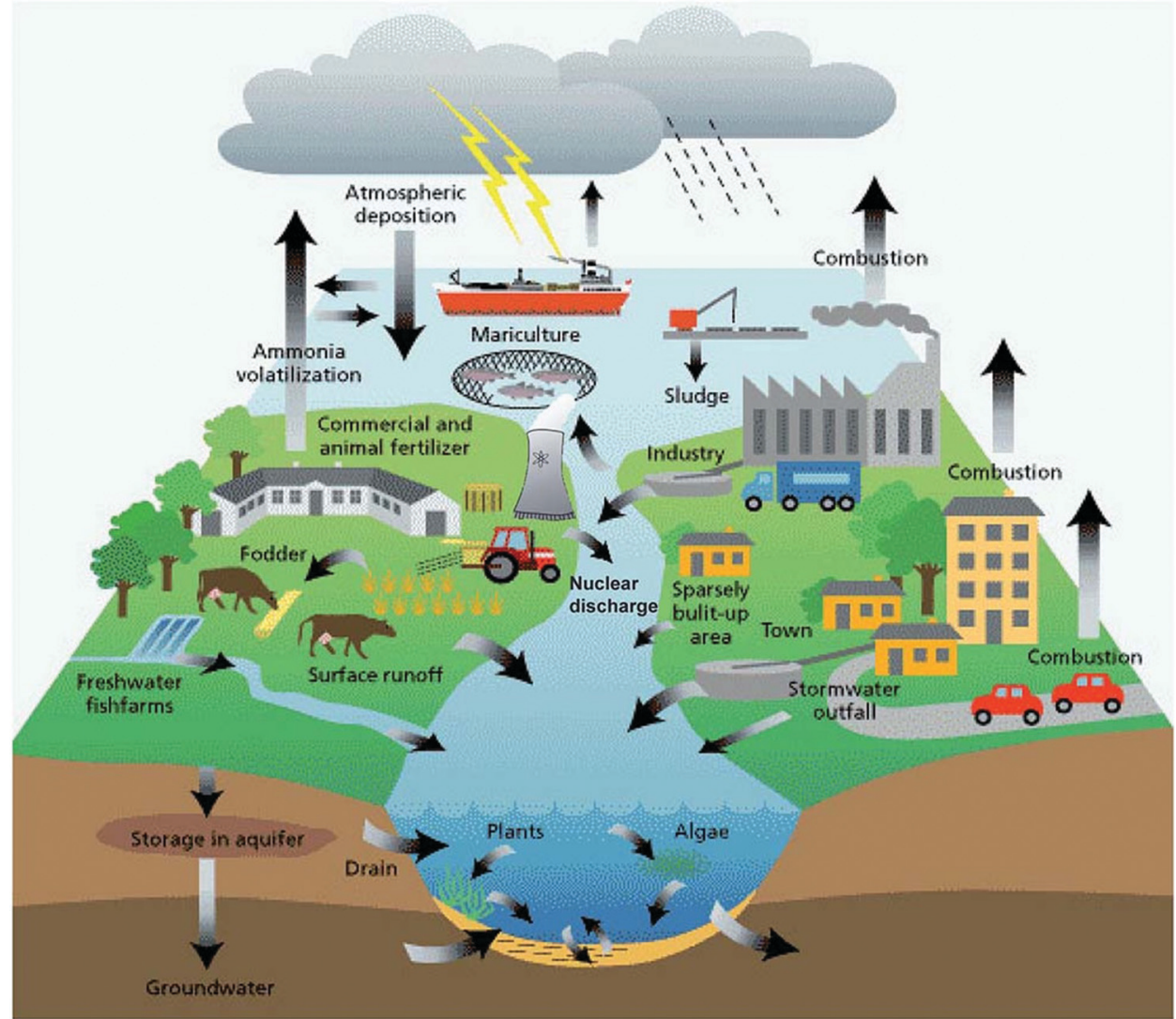

Fig. 1 Sources of groundwater contamination in the hydrologic cycle. ${ }^{4}$ Reproduced from ref. 4 with permission from the Royal Society of Chemistry.

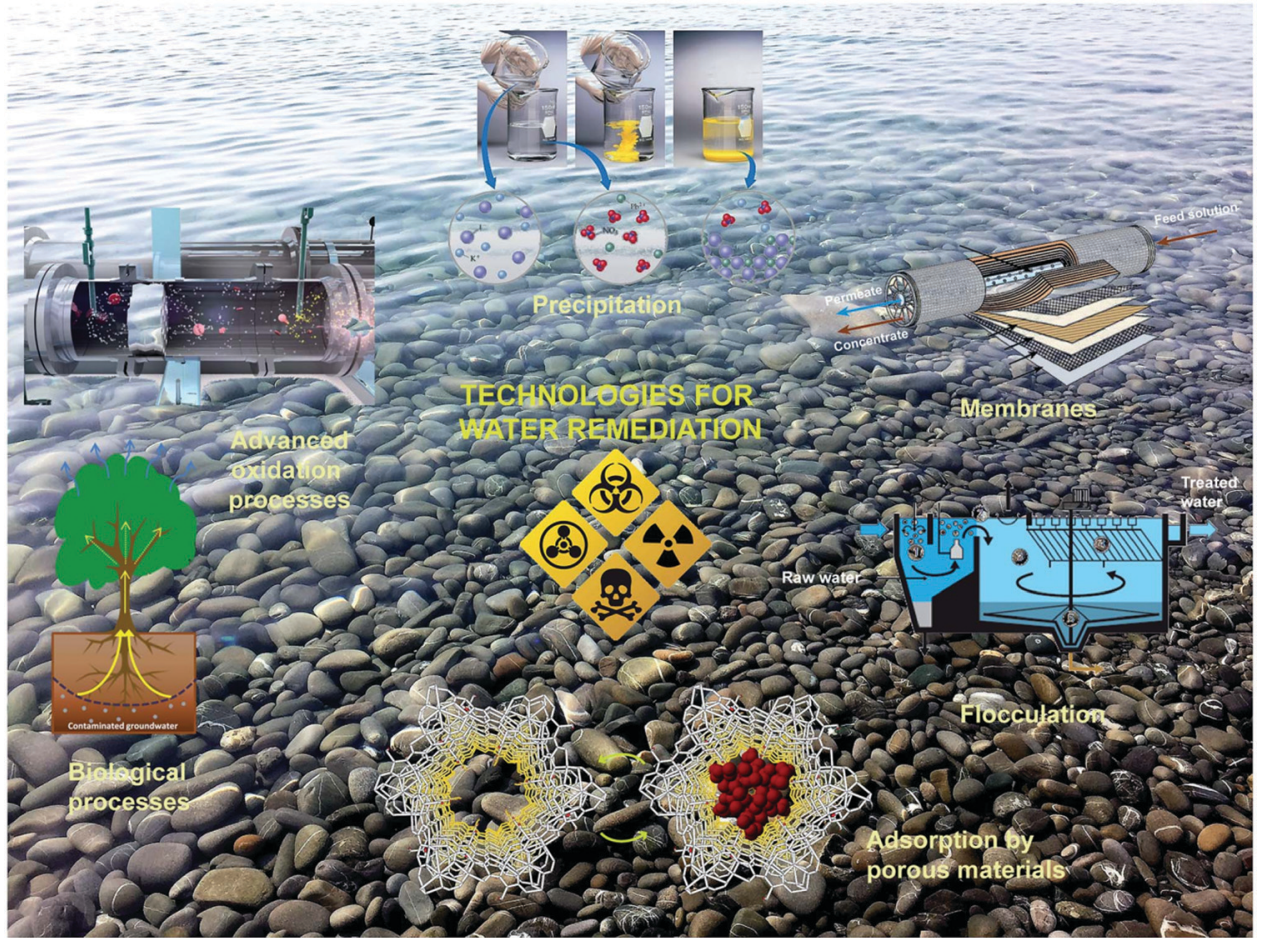

Fig. 2 Current technologies and processes for water decontamination. ${ }^{4}$ Reproduced from ref. 4 with permission from the Royal Society of Chemistry. 


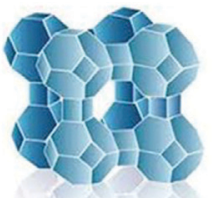

(a)

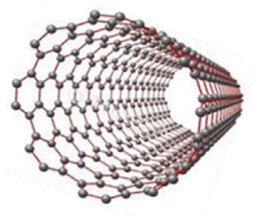

(d)

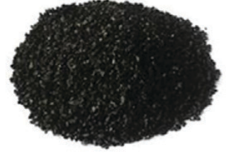

(b)

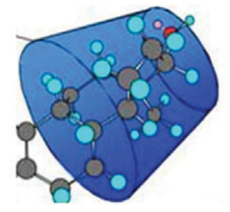

(e)

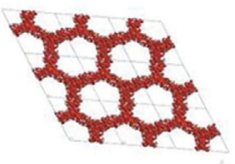

(c)

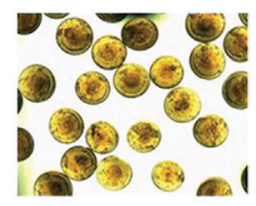

(f)
Fig. 3 Different types of adsorbents used for the removal of ECs: (a) zeolites, (b) Activated carbon, (c) mesoporous silica, (d) carbon nanotubes, (e) cyclodextrins, and (f) chitosan beads. ${ }^{31}$ Reproduced from ref. 31 with permission from Elsevier.

chemical technology is that it needs an ample amount of chemicals and electric energy. ${ }^{23}$ Recently, the advancement in the field of advanced oxidation has gained much attention because of its tremendous oxidizing property. However, although this technique is quite impressive, it is costly, and the generation of oxide intermediates is toxic to organisms. ${ }^{24,25}$ Thus, the abovementioned reasons make it clear that these methods are economically unpleasant. In the physical method, the traditional techniques used include membrane separation and adsorption technologies. The process of membrane separation is very effective, which includes nanofiltration, ultrafiltration, microfiltration, and reverses osmosis. ${ }^{26}$ However, membrane technology in wastewater remediation is not a standard technique, and although the use of membranes is economical, they can be polluted. ${ }^{27}$ Based on the literature, it can be presumed that the process of adsorption is the most regularly used technique for the remediation of dye wastewater due to its flexible and low-cost treatment and full effectiveness. ${ }^{28}$ Also, although there are many problems that need to be resolved, the adsorption mode does not form any hazardous materials ${ }^{29}$ (Tables 2 and 3).

Metal-organic frameworks (MOFs) are basically infinite crystalline structures composed of metal clusters bonded with organic ligands. $^{30,31}$ The characteristic feature of MOFs is that they are generally porous and flexible due to their structure and chemical tunability. MOF materials have also been used as adsorbents for gas-phase and liquid-phase separations. ${ }^{32}$ MOFs have also been employed in the adsorption of organics, ${ }^{33} \mathrm{H}_{2}{ }^{34}$ and $\mathrm{CO}_{2} \cdot{ }^{35-37}$ Recently, MOF materials showed good catalytic, and particularly, photocatalytic properties because their optical characteristics can be easily tuned to enhance their light harvesting properties, and thus employed as alternatives to other more photosensitive molecules. ${ }^{38-40}$ However, coordination compounds and most MOFs are potentially unstable in aqueous media. The stability of MOFs can be tuned in water after understanding the coordination between their metal and organic ligands. Over the years, great advancement have been achieved in the design of water-stable MOFs, which is a new class. This class of MOF materials can be immersed in water for a long period and at different $\mathrm{pH} .{ }^{41}$ Thus, MOFs are promising candidates for the treatment of wastewater due to their high

Table 1 The advantages and disadvantages of different dye removal technologies



Table 2 Adsorption of veterinary drugs over MOFs from aquatic systems

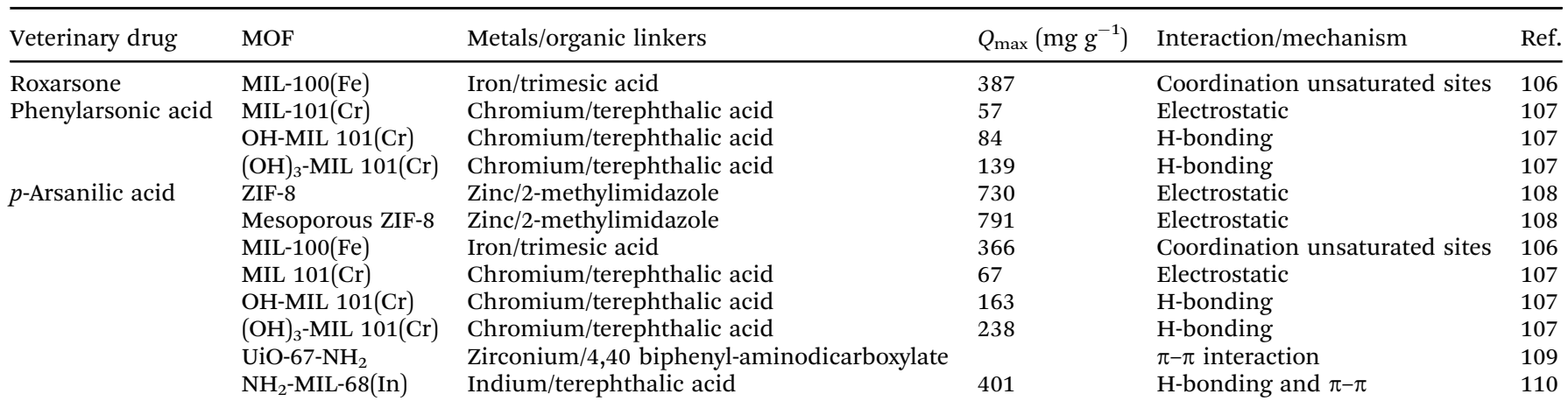


Table 3 Recently reported adsorption capacities of clays, zeolites and their composites

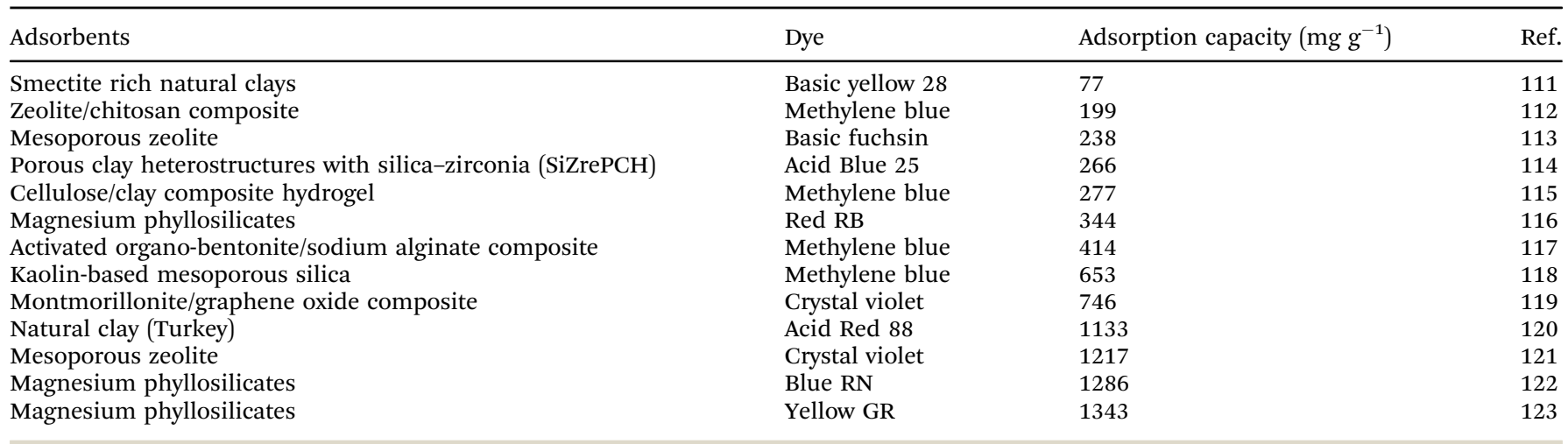

porosity and specific interactions between adsorbates and adsorbents. Moreover, their catalytic behavior can be tuned for the degradation of organic pollutants. Thus, in this review, we mainly discus the recent advancements in different topological and structural MOFs as good agents for dye adsorption and photocatalytic degradation of organic dyes. Research in the area of optimizing, modifying and introducing defects in MOFs can improve their adsorption performance. The optimized MOF materials having novel features such as one-dimensional (1D) linear molecules, two-dimensional coordination polymeric (2D) sheets and three-dimensional metal organic systems (3D) have attracted significant consideration. Mixed-metal complexes with functionalized linkers are the major forms of the modified MOFs. Defective MOFs are also involved in the formation of defect active sites. MOF-based composites obtained from the arrangement of MOFs with other functional materials have also been designed to enhance their surface area, adsorption capability and straightforward separation. The direct carbonization of MOFs as raw materials yields derived MOFs. Accordingly, we hope that MOFbased materials will be better used for the adsorption of organic pollutants and new prospects in this direction featuring their structure-activity relationship based on structural modifications should be elaborated by coordination chemists to enhance the material importance of MOFs.

In this review, the characteristic features of metal organic frameworks (MOFs) and their structural tuning triggering the capture of hazardous organic contaminants especially organic dyes are elaborated. This review features the types of adsorption, together with their mechanism and the factors promoting dye adsorption by a particular MOF. Our desire to work on functional materials especially MOFs and their dye adsorption properties led some important conclusions, which spurred us write this review as an informative paper to readers and scientists working in the field of dye adsorption.

\section{Metal organic frameworks (MOFs): structural features and types}

Hybrid materials consisting of organic and inorganic solids have an infinite and consistent crystalline nature created by metal ions or clusters with organic ligands. ${ }^{42}$ In 1995 , the first MOFs having permanent porosity were described. ${ }^{43}$ At that time, there was no conventional nomenclature introduced for this class of molecules, and thus there were numerous names in practice, such as microporous coordination polymers, ${ }^{44}$ porous coordination polymers, ${ }^{45}$ and zeolite-like MOFs. ${ }^{46}$ In an MOF, the organic linker and metal ions are linked through coordination bonds. The metal ion can be present in cluster form with organic linkers in multidentate organic ligands such as carboxylates and other organic anions such as sulfonate heterocyclic and phosphonate compounds. ${ }^{47}$ Recently, MOF-based materials have attracted great attention from researchers due to their versatile and potential applications. ${ }^{48,49}$ The characteristics features of MOFs that make them so popular are: (1) they are easy to synthesize compared with zeolites, (2) they can easily expand their surface area with tunable porosities having coordinative unsaturated sites, ${ }^{50}$ and (3) their ability to function without breaking their framework. ${ }^{51-54}$ The most interesting feature of MOFs is that they can be easily synthesized with diverse topologies, unusual structures and neutral skeletons. The first parameter to observe in the synthesis of MOFs is their solvent. The solvent acts as the first template, which is favorably correlated in the construction of zeolite-based materials, in which organic or inorganic templates are normally needed. Additionally, the solvent has limited interaction with the framework, which is an essential parameter for understanding the final framework with accessible pores, at comparatively low temperatures. On the other hand, the various metal ions in their mono-, di-, tri-, and tetravalent form can coordinate with the organic linker, whereas in traditional zeolites, only a small number of cations (such as $\mathrm{P}, \mathrm{Si}$ and $\mathrm{Al}$ ) can form these architectures. Thus, MOFs can be easily tuned to obtain a desired product with an improved functional application. The surface area, pore size and shape of MOFs can be easily tuned from the microporous to mesoporous scale. ${ }^{55,56}$ For instance, three MOFs comprising a zirconium metal ion, namely, UiO-66 (Zr-benzene dicarboxylate, Zr-BDC), UiO-68 (Zr-terphenyldicarboxylate, Zr-TPDC) and UiO-67 (Zr-biphenyldicarboxylate, Zr-BPDC) possessed surface areas of 1187, 4170 and $3000 \mathrm{~m}^{2} \mathrm{~g}^{-1}$, respectively. The different organic linkers ${ }^{56}$ with different metal ions or clusters in MOFs form different frameworks, having diverse 
topological and physicochemical features. The CUSs or OMSs, stability, and Lewis acidities and their reaction with related organic linkers $^{55}$ such as CPO-27 (Me-DHTP) (2,5-dihydroxy terephthalate), where $\mathrm{Me}=\mathrm{Co}^{2+}, \mathrm{Ni}^{2+}, \mathrm{Fe}^{2+}, \mathrm{Mn}^{2+}$, and $\mathrm{Zn}^{2+},{ }^{2-59}$ MIL-100 (Me-BTC (benzenetricarboxylate), where $\mathrm{Me}=\mathrm{Fe}^{3+} \mathrm{Cr}^{3+}$, and $\mathrm{Al}^{3+}$ ), ${ }^{60-62}$ and MIL-96 (Me-BTC; $\mathrm{Al}^{3+}, \mathrm{Cr}^{3+}, \mathrm{Ga}^{3+}$, and $\left.\mathrm{In}^{3+}\right) .{ }^{63-66}$ After the formation of crystalline materials, the physicochemical properties of MOFs can be easily tuned. ${ }^{67-70}$ Various reported MOFs have a robust nature, and their porous quality allows their post-synthetic modification (PSM) without affecting their original structure and topology. ${ }^{67-70}$ Various techniques can be adopted to anchor the effect of acid or base on MOFs via PSM, i.e., postfunctionalization of amine groups with sulfones, ${ }^{70}$ post-synthetic framework sulfonation, ${ }^{69}$ grafting of cysteamine or ethylenediamine $(\mathrm{ED})^{70}$ and amendment with different acid anhydrides. ${ }^{71}$ On the other hand, there are various ways in which MOFs can be easily tuned by loading active metals (such as $\mathrm{Pd}, \mathrm{Cu}$ and $\mathrm{Ag}$ ) or by forming composites. ${ }^{72}$ Further, the thermal and mechanical stability of MOFs are crucial and much attention has been given to the stability of MOFs in aqueous media. ${ }^{73}$ The common limitation for the utilization of MOFs in numerous applications is their instability in water both in the liquid and vapor phase. ${ }^{74}$ Numerous MOFs such as MOF- 5 and MIL-101-V can be degraded through ligand displacement or hydrolysis, which has been confirmed by multiple experimental and computational investigations. $^{75}$ On the other hand, MOFs (MIL-100) (Al, Cr, and Fe) ${ }^{76,77}$ MIL-101-Cr, ${ }^{78}$ and UiO-66, ZIF- $8^{79}$ with high water stability and can be employed for water purification through the adsorption process. Various techniques such as composite formation, ${ }^{80}$ incorporating water repellent functional groups, ${ }^{81}$ and fluorination ${ }^{82}$ can be used for enhancing the stability of
MOFs in aqueous media. In last few decades, MOFs have been widely developed due to their exceptional chemistry and unusual characteristics, and their great potential applications in diverse research areas (Fig. 4). ${ }^{83}$ Finally, MOFs have been recognized as suitable substances for the liquid-phase adsorption of different compounds, including the compounds having sulfur ${ }^{84}$ and nitrogen, ${ }^{85}$ in different media. This review focuses on the MOF-based adsorptive removal of hazardous compounds, particularly from aqueous media.

The issue of water pollution has gained much consideration due to the industrial expansion globally, which has become the principal threat to the environment. ${ }^{86-88}$ Heavy metal ions and organic pollutants are mainly responsible for water pollution. ${ }^{86-92}$ A huge amount of organic pollutants is present in natural water, which pose a risk to living bodies and also make purifying wastewater difficult (Table 4). ${ }^{89}$ The United States Environmental Protection Agency has listed the poisonous organic pollutants that should be separated from wastewater before it is released into the atmosphere. ${ }^{90}$ The list is comprised of organic compounds such as benzene, benzenes (chlorinated), phenols (chlorinated phenols), nitrobenzene and phthalates, which are used in very toxic industries and threaten human life and the environment. ${ }^{91-95}$ Furthermore, it contains various hazardous materials such as dyes, spilled oils, and pharmaceuticals and personal care products (PPCPs). ${ }^{86-90}$ Nowadays, the most critical threat to the environment is dye molecules because of their organic nature and stability in aqueous medium, making them tough to degrade. Essentially, dye materials are used in the plastic, textile, painting, leather, and paper industries. Recently, it was reported that approximately 100000 dyes are produced every year and 2\% of them released into the atmosphere. ${ }^{91}$ Regularly, synthetic dyes are

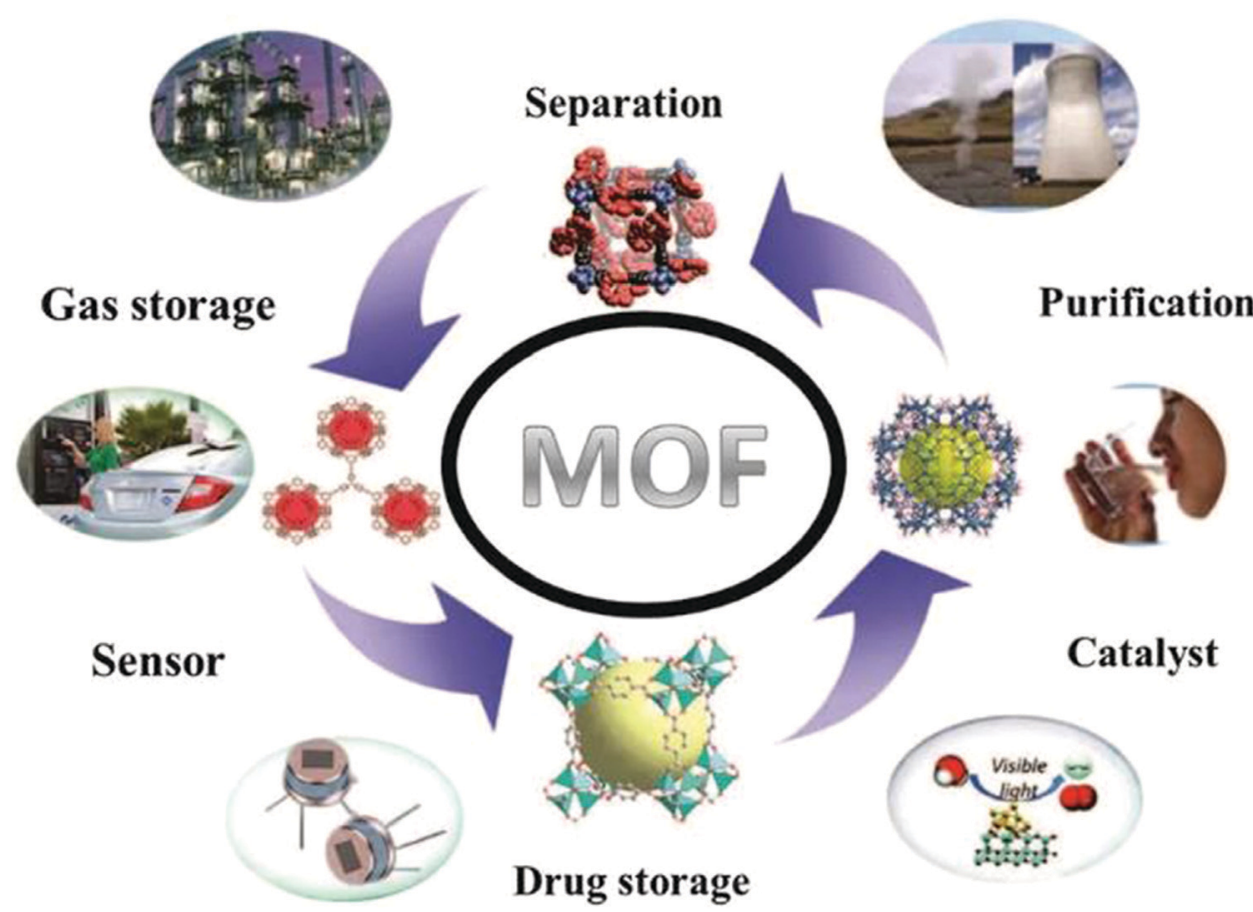

Fig. 4 Wide-ranging applications of MOFs. ${ }^{49}$ Reproduced from ref. 49 with permission from the Royal Society of Chemistry. 
Table 4 Classification, sources and hazards of PTS

\begin{tabular}{|c|c|c|}
\hline PTS & Sources & Hazards \\
\hline Synthetic dyes & $\begin{array}{l}\text { Food industry, dye wastewater, papermaking } \\
\text { wastewater, textile and printing wastewater }\end{array}$ & High chromaticity, carcinogenesis, and toxicity \\
\hline Plasticizers & Chemical industry and plastics industry & $\begin{array}{l}\text { Inhibition of human central nervous system, } \\
\text { and strong stability }\end{array}$ \\
\hline $\begin{array}{l}\text { Polycyclic aromatic } \\
\text { hydrocarbons (PAHs) }\end{array}$ & Petrochemical industry and coking industry & Strong stability and strong carcinogenicity \\
\hline Organic cyanogen compounds & $\begin{array}{l}\text { Petrochemical industry, artificial fiber industry, } \\
\text { and coking industry }\end{array}$ & Acute toxicity \\
\hline Heterocyclic compounds & Heterocyclic organisms & $\begin{array}{l}\text { Carcinogenesis, Strong stability, Mutagenicity, } \\
\text { and Bioconcentration }\end{array}$ \\
\hline Polychlorinated biphenyl (PCB) & $\begin{array}{l}\text { Lubricating oil industry, chemical wastewater, } \\
\text { mechanical industry, and plastics industry }\end{array}$ & Acute toxicity and carcinogenesis \\
\hline Synthetic detergents & $\begin{array}{l}\text { Food industry, textile industry, tannery industry, } \\
\text { and papermaking industry }\end{array}$ & $\begin{array}{l}\text { Solubilization of carcinogenic polycyclic aromatic } \\
\text { hydrocarbons }\end{array}$ \\
\hline
\end{tabular}

carcinogenic and teratogenic in nature when flashed to the aquatic system, and thus may cause severe diseases such as acute or chronic disease. ${ }^{92}$ These impurities in water are very difficult to degrade because they absorb sunlight. ${ }^{94,95}$ Currently, PPCPs are also considered as contaminants, which consist of the chemical materials used in cosmetics, veterinary drugs, agrochemicals, medicines, fragrances, fungicides, and disinfectants. ${ }^{96-98}$ Due to their stability, they are tough to degrade or convert in wastewater processing plant, and thus enter the aquatic system. ${ }^{96}$ The discovery of PPCPs in the atmosphere was investigated in the late $1980 \mathrm{~s} .{ }^{97,98}$ A previous study suggested that the liquid waste from water treatment plants, lakes, rivers, and occasionally groundwater and their demand increase with time. ${ }^{99}$ Meeting the requirements of customers, PPCPs have long shelflives, and thus endure in the atmosphere even after the product has been used. ${ }^{98,100,101}$ However, the effects of PPCPs on living organisms have rarely been investigated, although it has been documented that these poisonous pollutants can cause endocrine disruptions and further change in hormonal effects. ${ }^{102,103}$ As in known, in oil spills, the liquid is released into the environment unintentionally due to anthropogenic actions, together with storage, transporting, drilling and manufacturing. Presently, oil spills are very common, for example the Korean (2007, Yellow Sea), Deepwater Horizon (2010, Gulf of Mexico), and Mayflower (2013) oil spills. Due to the severe effect of oil spills on the marine and aquatic systems, during the Deepwater Horizon oil spill, many marine animals died, spanning an area of around $1300 \mathrm{~km}$ of beaches and shoreline. ${ }^{104}$ The investigation into numerous terrible oil spills and their impact on the atmosphere has spurred researcher to design ecological and effective methods for the remediation of wastewater. ${ }^{105}$ Organic dyes, which are organic moieties and can be easily found in wastewater, are very dangerous to human health (Fig. 5). Different dyes and their structures and absorption peaks are listed in Table 5. Metal organic frameworks (MOFs) or coordination polymers (CPs) have been employed recently for the adsorption of these dyes in aqueous media. This new class of organic-inorganic porous materials have a high surface area, tunable pore geometry, and different topological properties, making them a suitable candidates for the treatment of wastewater through dye adsorption ${ }^{106-123}$ (Tables 2 and 3).

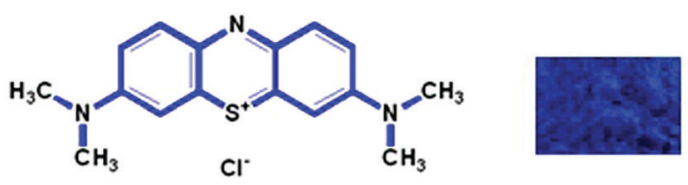

(a)<smiles>CN(C)C1CCC(N=NC2CCC(S(=O)(=O)[O-])CC2)CC1</smiles>

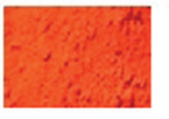

(b)

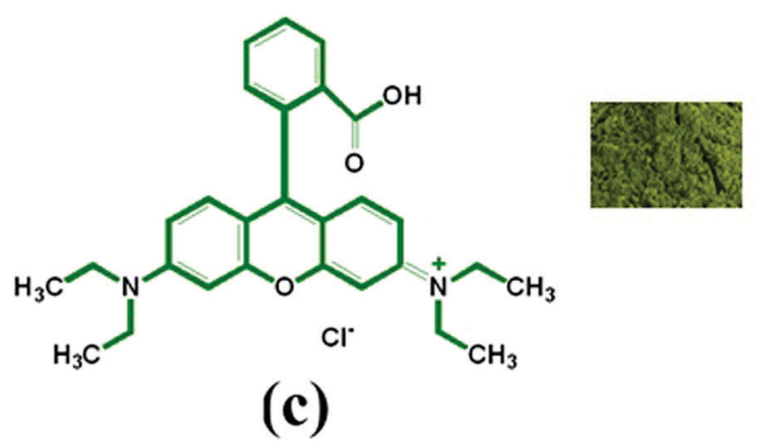

Fig. 5 Powdered forms of some hazardous aromatic dyes: MB (a), MO (b) and $\mathrm{Rh}-\mathrm{B}$ (c) and their structures.

\section{Characteristics of MOFs as adsorbents}

In the last few decades, there many studies have been performed in the field of designing adsorbent materials for wastewater remediation. Accordingly, metal-organic frameworks (MOFs) have been reported as solid adsorbent materials due to their high stability. Furthermore, MOFs exhibit some added peculiarities that make them promising candidates for the 
Table 5 The classifications, chemical structures and absorption wavelengths of some commonly used organic dyes

Name of dye

Methylene blue (MB)

Methyl orange (MO)

Rhodamine B (RhB)

Methyl violet

Alizarin

Crystal violet (CV)

Congo red (CR)

\section{Chemical structure}<smiles></smiles>

$\mathrm{Cr}$<smiles>CN(C)c1ccc(/N=N/c2ccc(S(=O)(=O)[O-])cc2)cc1</smiles><smiles>CCN(CC)c1ccc2c(-c3ccccc3C(=O)O)c3ccc(=[N+](CC)CC)cc-3oc2c1</smiles><smiles>CN(C)c1ccc(C(=C2C=CC(=[Cl+])C=C2)c2ccc(N(C)C)cc2)cc1</smiles><smiles>O=C1c2ccccc2C(=O)c2c1ccc(O)c2O</smiles><smiles>CN(C)c1ccc([C](c2ccc(N(C)C)cc2)c2ccc(N(C)C)cc2)cc1</smiles><smiles>Nc1c(/N=N/c2ccc(-c3ccc(/N=N/c4cc(S(=O)(=O)O[Na])c5ccccc5c4N)cc3)cc2)cc(S(=O)(=O)[O-])c2ccccc12</smiles>

Nature

Absorption $\lambda_{\text {max }}(\mathrm{nm})$

Cationic

664

Anionic

467

Cationic

552

Cationic

Variable

Neutral Variable

Cationic Variable

Anionic Variable 
Table 5 (continued)

Name of dye Chemical structure

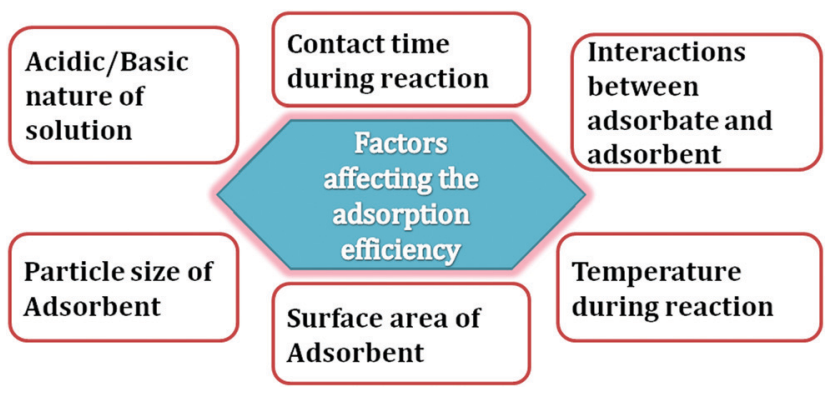

Fig. 6 Factors affecting the adsorption process using MOFs as adsorbents

adsorption process (Fig. 6). The main features of MOFs are their extensive surface area, porosity, high thermal and mechanical stability, and excellent functionality. For an adsorbent, a wide surface area and especially high porosity make it very efficient because the adsorption sites become accessible and the diffusion of hazardous substances can occur easier. Accordingly, MOFs can be constructed with better porosity, where the pores of the material are guaranteed due to the crystallinity of MOFs. Synthesized MOFs with better porosity can be easily tuned by changing the organic spacers and their coordination with different metal ions. Currently, the in situ adjustment of the post-synthetic structure of MOFs has attracted much interest because it does not change the internal topology of the MOFs. ${ }^{124}$ The tuning of the topology and properties of MOFs can be easily done simultaneously with their organic and inorganic components. Furthermore, their pore size and chemical environment may trigger synergy between the adsorbates and adsorbent. Additionally, the spontaneous synthesis, large surface area, functionalized organic linkers and thermal stability of MOF make them better candidates than regular adsorbents compounds, e.g., carbonaceous solid, zeolites and silica. The pores in MOFs are effective space for the adsorption of guest molecules, which depends on the structural characteristics of the MOF. Ultimately, the unique features, wide range of structural qualities and their inorganic-organic hybrid features of MOFs make them beneficial for efficiently adsorbing hazardous materials. Recently, M. Shahid et al., reported a porous metal organic framework, $[\mathrm{Cu}(\text { Metet })]_{n}$ (MOF-1), which was synthesized via a solvothermal method employing $\mathrm{NaN}_{3}$ and 2-amino-4-picoline. ${ }^{125}$ The porous MOF, which was formed in situ during the autoclave, reaction showed a tremendous adsorption performance towards methylene blue dye. The adsorption process was

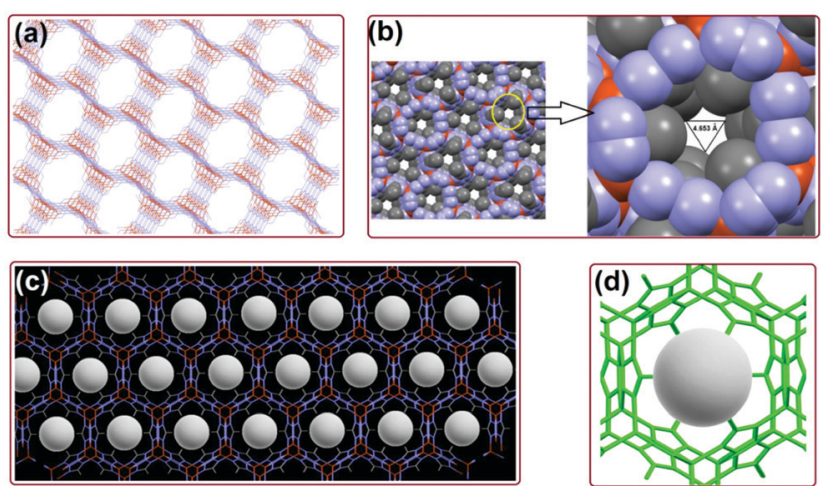

Fig. 7 (a) Expanded 3D model of MOF-1. (b) Space filled model of MOF-1 showing a single unit of the expanded MOF-1. (c) and (d) Space occupied by the MB structure in the 3D MOF-1. ${ }^{125}$ Reproduced from ref. 125 with permission from Springer.

carried by adsorbing the dye into the pores of MOF-1, as shown in Fig. 7.

Although it is evident from the above discussion on the structural features of MOFs that they are better candidates for various functional applications, there are some specific requirements in the structure of their framework that cause the dye adsorption to occur effectively. Porosity is the most common factor according to the size of the dye, e.g., linear dyes such as methylene blue easily enter the porous network, thus enhancing the adsorption phenomenon. However, not only porous materials, but also non-porous MOFs have been found to absorb dyes owing to their special structural features such as the presence of suitable free functional groups like $-\mathrm{NH}_{2}$ and $-\mathrm{OH}$, which form stronger H-bonding interactions with dye molecules. The HSAB principle has also been applied in MOFs for interaction of hard donor sites such as nitrogen with their hard functional groups, thus providing hard-hard (ionic) interactions. Also, the planar aromatic rings present in the skeleton of MOFs are an added advantage for the interaction of dye molecules through $\pi-\pi$ and $\mathrm{C}-\mathrm{H} \cdots \pi$ interactions. ${ }^{126}$ Numerous other suitable functions can also be used to consolidate the binding of dye molecules on the surface of MOFs through various non-covalent interactions.

To enhance the adsorption process, the dimensionality of MOFs also plays a key role. Thus, the synthetic method to obtain MOFs with suitable dimensionality is also important to enhance the adsorption process. Specifically, 3D (porous or non-porous materials) followed by 2D MOFs are suitable candidates depending on the reactants and the reaction type 
and conditions. Thus, when considering the synthesis of MOFs for dye adsorption applications, these factors should be the focus when choosing reactants to trigger the effective adsorption of dye molecules by the resulting product. Therefore, a proper understanding of the structural features and their tuning becomes necessary in designing adsorbent MOFs.

\subsection{Stability of MOFs in water}

The water stability of MOFs is essential for their adsorption of organic pollutants. MOFs have been applied in diverse fields, i.e., catalysis, gas adsorption and separation, where their water stability is not necessary, unlike the adsorption process (Fig. 8). Thus, the feeble stability of MOFs in water restricts their application as adsorbents for the treatment of wastewater. ${ }^{127}$ The synthesis of water-stable MOFs is challenging, but several articles on water-stable MOFs such as MOF-74, MIL-101 (Cr, Fe, Al), ZIF-8, and MIL-53 (Cr, Al) have been published. ${ }^{128}$ The deficiency in the water stability of MOFs is due to the weak bonding between their metal ions and organic spacers. The functionality of several MOFs has been observed in varying humid atmospheres such as water vapor, water, and acid/base aqueous media. By following the characteristic features of a series of different MOFs, implementing high-throughput steam treatment demonstrated that the stability of MOFs can be efficiently tuned via the formation of metal and organic ligand coordination bonds and modifying the oxidation state of the metal ions. ${ }^{127}$ Accordingly, MOFs such as MOF-74 (Mg-O bond), ZIF-8 (Zn-N bond), Cr-MIL-101 (Cr-O bond) and Al-MIL-53 (Al-O bond) have been seen to exhibit sufficient stability in contrast to that of $\mathrm{Cu}-\mathrm{O}$ (HKUST-1) and $\mathrm{Zn}-\mathrm{O}$ (MOF-5 and MOF-508). ${ }^{127}$ Various studies have demonstrated that ZIF-8 MIL-101 and MIL-100(Fe) are stable in aqueous media. An additional study by Schoenecker et al., on water vapor adsorption suggested that the MOFs HKUST-1, Mg MOF-74, and UiO$66\left(-\mathrm{NH}_{2}\right)$ remained stable, while DMOF-1, DMOF-1-NH UMCM-1 were unstable under 90\% relative humidity. ${ }^{129}$ According to the observations to date, the zirconium MOFs, i.e., UiO-66 and UiO-66- $-\mathrm{NH}_{2}$, are the stable MOFs in the water. An MOF (CAU-10-H) employing aluminum hydroxide isophthalate was studied under humid multi-cycling conditions, and it was observed that it was remarkably stable. ${ }^{130}$ The properties of

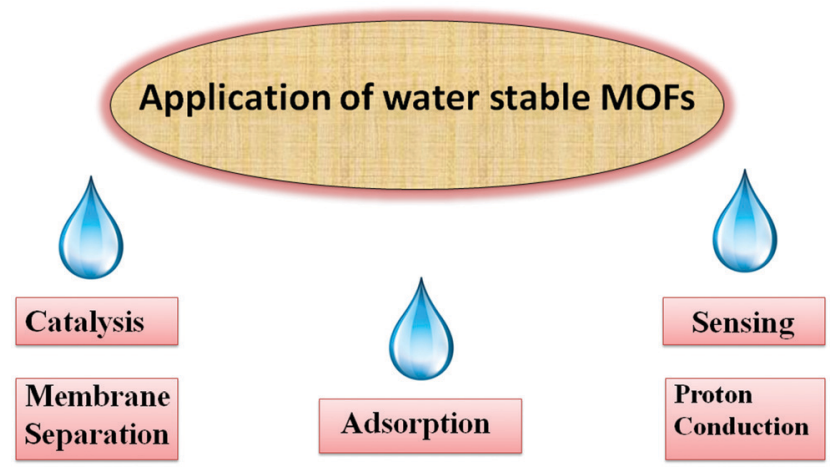

Fig. 8 Various applications of water-stable MOFs. different MOFs have been investigated in water. For instance, two zinc-based MOFs such as MOF-177 and MOF-5 has been observed to be moisture sensitive. Due to the hydrolysis of the framework, the porosity and surface area of the MOFs were reduced. ${ }^{131}$ The investigation by Greathouse and Allendorf using molecular dynamic simulation also suggests that MOF-5 is unstable. ${ }^{132}$ Another MOF reported by DeCoste et al., underwent complete distortion after one-day exposure to water, and third material was named Mg-MOF-74. ${ }^{133}$ The identical decomposition of the framework of HKUST-1 was observed when it was exposed to $90 \%$ humidity under ambient conditions. Further, Cychosz et al., carried out PXRD analysis to justify the stability of various MOFs, including UMCM-150, MOF-177, ZIF-8, MOF-505, MOF-5, HKUST-1 and MIL-100 (Cr), in aqueous media, and observed that among them, MIL-100 (Cr) and ZIF-8 were stable in pure water solution for up to 1 month. ${ }^{134}$ The excellent stability of MOFs of chromium is because of the inertness of the chromium metal ion. ${ }^{134,135}$ On the other hand, due to the higher coordination sites of their secondary building units, MIL-125 and UiO-66 were observed to have better stability. ${ }^{136}$ Individual MOFs were also investigated for their stability in acidic or basic medium or buffer medium. Zirconium-based MOFs such as PCN 224 and 222 were stable, even under harsh conditions. ${ }^{137,138}$ These MOFs exhibited significant stability in acidic and basic medium (pH 1-11). ${ }^{137,138}$ On the contrary, Al-based MOFs are structurally stable in aqueous medium, but some of them dissolve in acidic conditions. ${ }^{139}$ The durability of $\mathrm{V}, \mathrm{Cr}$, and Al-BDC have also been investigated at room temperature, and among them, Cr-BDC was established to be the most stable. ${ }^{140}$ Besides, Kandiah et al., investigated the effect of acid and base on some Zr-based MOFs, and all of them maintained their stability in aqueous medium under acidic conditions $(\mathrm{pH}=1)$. On the other hand, in $\mathrm{NaOH}$ solution $(\mathrm{pH}=14)$, MOF UiO-66- $\mathrm{NO}_{2}$ retained its structural identity, while other MOFs such as UiO-66 and UiO-66-Br inappreciably changed their identity within only $2 \mathrm{~h}$ at $\mathrm{pH}=14 .{ }^{141}$ Park et al., described the stability of ZIFs in water, aqueous sodium hydroxide, benzene and methanol for almost a week at different temperatures, which were found to be stable. ZIF-8 maintained its composition for seven days in all the warm organic solvents and water for up to $24 \mathrm{~h}$ in $0.1 \mathrm{M}$ and $8 \mathrm{M} \mathrm{NaOH}$ at $100{ }^{\circ} \mathrm{C} .{ }^{142}$ Furthermore, the stability of MOF materials in buffer medium is also an essential parameter, which has been reported in a few articles. In phosphate buffer solution, Cunha et al., investigated the stability of seven carboxylate-based MOFs at $7.4 \mathrm{pH}$ and at $37^{\circ} \mathrm{C}$. The stability of the MOFs followed the order of Fe-MIL-100/-127 > Fe-MIL-53, UiO-66- $\mathrm{NH}_{2}>$ Fe-MIL-53-Br UiO-66 > UiO-66-Br. ${ }^{143}$ Jung et al., explained the stability of IRMOF-3 in PBS buffer at a pH value of 7.3. Powder XRD was employed to investigate the stability of IRMOF-3 after $1 \mathrm{~h}$ incubation in the buffer, and it was found that an increase in the incubation time $(6 \mathrm{~h})$ modified its XRD pattern. ${ }^{144}$ Understanding the stability of MOFs in aqueous media is imperative for the formation of water stable and water sensitive MOFs for practical utilization. Distinct theoretical studies have also been conducted, such as molecular dynamics and quantum mechanical calculations, to provide knowledge about the interaction of MOFs with water. ${ }^{132,145,146}$ MOF-5 can be readily 
protonated due to the appearance of water in its formation, where the oxygen in the water molecule attacks the tetrahedral $\mathrm{ZnO}$, and frees its linker. ${ }^{131,132}$ De Toni recommended that the development of a water cluster at the $\mathrm{Zn}_{4} \mathrm{O}$ section is due to the displacement of a ligand, which maintains the water coordination in the MOF structure. ${ }^{146}$ An accurate study of the hydrolysis of MOF-74, IRMOF-1 and IRMOF-10 utilizing reactive force field investigation was performed, ${ }^{145}$ in which it was assumed that when water molecules are introduced in the framework of IRMOF-1, they attack the $\mathrm{Zn}-\mathrm{O}$ moiety. The water molecules dissociate into $\mathrm{H}^{+}$ and $\mathrm{OH}^{-}$, and subsequently the $\mathrm{OH}^{-}$anion coordinates with the $\mathrm{Zn}$ site, while $\mathrm{H}^{+}$coordinates with the organic part, causing the structure to become distorted and deflated. ${ }^{145}$ Low et al. so estimated with the help of computational and experimental studies that ligand displacement and hydrolysis are the principal causes of the low stability of MOFs in water. ${ }^{127}$ There are several review article on the degradation mechanism of MOFs in aqueous media. ${ }^{126,147,148}$ To obtain MOFs with excellent stability, different procedures have been reported, such as ligand fictionalization, metal cation exchange, and hydrophobicity enhancement. ${ }^{149}$ The diffusion of water protective groups on the ligand is the most accepted method for increasing the stability of MOFs. ${ }^{150,151}$ The synthesis of Banasorb-22 by $\mathrm{Wu}$ et al., with a water protecting group on the organic linker endowed the MOF with tremendous sorption capability and better stability. ${ }^{152}$ By applying a tetraethyl1,3,6,8-pyrenetetraphosphonate linker, which has a good monoester phosphonate ligand, a new porous barium-based MOF material named CALF-25 was synthesized, exhibiting hydrophobicity due to the presence of ethyl ester groups. ${ }^{150}$ MOFs based on naphthyl, anthracene, and tetramethyl-BDC preserve their crystalline form even after water exposure. ${ }^{153}$ By varying the organic linkers, different chemically stable MOFs can be designed, such as PCN-600 (M), where $\mathrm{M}=\mathrm{Mn}, \mathrm{Fe}, \mathrm{Co}, \mathrm{Ni}$ and $\mathrm{Cu}$ derived with a porphyrin ring as the organic ligand. ${ }^{154}$ Wang et al., elaborated two water-stable MOFs, which were isostructural, using the flexible ligand $\left[\mathrm{Zr}_{6} \mathrm{O}_{4}(\mathrm{OH})_{8}\left(\mathrm{H}_{2} \mathrm{O}\right)_{4}(\mathrm{CTTA})_{8 / 3}\right]$ $\left(\right.$ BUT- $_{12}, \quad \mathrm{H}_{3}$ CTTA $=$ 50-(4-carboxyphenyl)-20,40,60-trimethyl[1,10:30,100-terphenyl]-4,400 dicarboxylic acid). ${ }^{155}$ These two MOFs showed very good efficiency in detecting particular antibiotics and organic explosives in aqueous media. ${ }^{155}$ The fundamental nature of the ligand plays a critical role in the stability of MOFs in aqueous media because the bond between metal-ligand becomes powerful. The hydrophobic Cu-MOF $\mathrm{Cu}_{2} \mathrm{~L}$, where $\mathrm{L}=3,30,5,50$-tetraethyl-4,40-bipyrazolate, has been reported, which showed tremendous thermal mechanical and water stability. ${ }^{156}$ The PXRD pattern of this MOF was examined in acidic and basic medium, and there were no changes in the intensity of its PXRD pattern after $24 \mathrm{~h}^{156}$ Similar MOF materials have also been synthesized, such as azolate-based MOFs with better stability in water than that based on the carboxylate ligand due to the basicity of the ligand. ${ }^{157,158}$ To further improve the water stability of MOFs in water, Li et al., incorporated the hydrophobic $\mathrm{C}_{60}$ in a c-cyclodextrin MOF and used it for drug delivery. ${ }^{159}$ The addition of different metals to MOF materials has gained significant attention due to the stronger metal-ligand bond, which enhances their water stability. A nickel-doped MOF-5 was synthesized by Li et al., which retained it crystallinity against humidity and possessed a higher surface area than that of its parent MOF-5. ${ }^{160}$ Catenated MOFs are also attracting attention due to their thermal stability and low water-loading in their frameworks. Chen et al., reported MOF-14, where 4,40,400 benzene-1,3,5-triyl-tribenzoic acid is the organic ligand with copper(II) nitrate in DMF solvent via a catenation process, which is quite robust in nature. MOF-14 was stable at room temperature, but exhibited low solubility in water and other organic solvents. ${ }^{161}$ Also, MOF-508 was constructed employing BTTB (4,40,400,4000-benzene-1,2,4,5tetrayltetrabenzoic acid) and a pillared ligand such as bipyridyl, which was stable up to $90 \%$ moisture due to the catenation in its framework. ${ }^{162}$ Further, a tetravalent zirconium-based network was designed, $\mathrm{Zr}_{6} \mathrm{O}_{4}(\mathrm{OH})_{4}\left(\mathrm{CO}_{2}\right)_{12}$ (UiO-66), which was stable in water. ${ }^{136}$ MOFs with $\mathrm{Zr}$ and $\mathrm{Cr}$ metal ions are quite robust in nature due to the higher coordination sites of their secondary unit and their inertness.

\subsection{Defective MOFs as adsorbents}

Defective MOFs have developed as an enthusiastic topic due to their promising strategy to improve the structure of MOFs to achieve a better adsorption process. ${ }^{162}$ A defect is a disturbance in the structure and heterogeneity of MOFs, which conclusively destroys the arrangement of the atoms and surface properties of the final MOFs. ${ }^{163}$ Various defects have been introduced in structure of MOFs such as acid modulators, mixed linkers and post-synthetic modification of MOFs with inorganic acids. The introduction of acetic acid in UiO-66 was investigated to enhance the adsorption process. The defective MOF showed a selective adsorption property with a positive zeta potential, large surface area and better adsorption capacity. ${ }^{164}$ The role of acetic acid in the modulation of UiO-66 was studied for the enhanced adsorption of dichloromethane. The adsorption capacity changed due to the missing linker defect, as demonstrated by the change in surface area from 980 to $1470 \mathrm{~m}^{2} \mathrm{~g}^{-1} \cdot 165$ The introduction of defects in MOFs makes them more porous in nature. ${ }^{166}$ The reaction of MIL-101 (Cr) crystals with a high molar ratio of formic acid/ $\mathrm{CrCl}_{3}$ resulted in a better shape, larger surface area and effective adsorption performance in the MOF. ${ }^{167-169}$ During the course of the synthesis, the addition of trifluoroacetic and $\mathrm{HCl}$ increased the adsorption capacity of the crystalline material. ${ }^{170}$ Thus, it can be concluded that defects increase the surface area, expand the pore size and enhance the chemical stability of MOFs. ${ }^{171}$ It can be seen in Fig. 9 that the change in the BET surface area of the MOFs is mainly due to acetic acid, benzoic acid and formic acid. ${ }^{172}$ Accordingly, a missing linker defect was synthesized using an acid modulator to construct an MOF with enhanced hydrophobicity, which provided various possibilities for the adsorption of dyes. ${ }^{173}$

\subsection{Functionalized MOFs as adsorbents}

MOF materials can be used as precursors for the synthesis of several nanostructure materials, e.g., heteroatom-doped carbons, transition metal oxide-carbon (TMO@C) composites and transition metal oxides (TMOs). ${ }^{174}$ Nanostructure-derived MOFs exhibit 


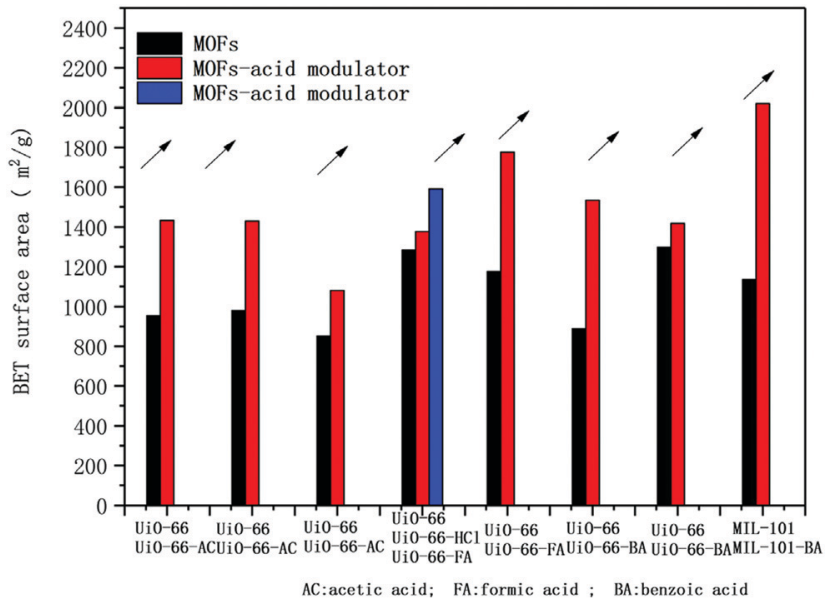

Fig. 9 Change in the BET surface area of MOFs based on modulator. ${ }^{172}$ Reproduced from ref. 172 with permission from Elsevier.

various special features as follows: (1) unique ability due to their chemical features compared to other MOFs; (2) expanded surface areas; and (3) inexpensive and easy to modify. For a better adsorption performance, MOF-5 was employed to obtain hierarchically porous carbon (HPC) via pyrolysis and graphitization. The HPC showed better adsorption of aromatic contaminants due to its characteristics feature and $\pi-\pi$ interactions. ${ }^{175}$ Further MOF1 was applied to derive HPC containing oxygen and nitrogen at $100{ }^{\circ} \mathrm{C}$, which resulted in the efficient adsorption of PPCPs. ${ }^{176}$ Using MAF-6, porous carbon was derived as a probable adsorbent for non-natural sweeteners due to its expanded porosity, surface functionality, hydrophobicity and thermal stability. The pyrolysis temperature is the critical factor to achieve enhanced adsorption, and $6 \mathrm{~h}$ was considered a suitable time. ${ }^{177}$ Additionally, ZIF-8 templated with carbons via the polymerization of ZIF-8 using furfuryl alcohol (FA) and a supplementary carbon source resulted in an excellent adsorption performance. ${ }^{178}$ ZIF-8 was also employed as a precursor because of its high thermal stability, sodalite-like structure, and mild product conditions. ${ }^{179}$ Further, the carbonated ZIF-8 was employed as an adsorbent at $100{ }^{\circ} \mathrm{C}$, which exhibited a better adsorption performance toward $\mathrm{MB}$ (186.3 $\mathrm{mg} \mathrm{g}^{-1}$ ) compared with that of ZIF-8 $\left(19.5 \mathrm{mg} \mathrm{g}^{-1}\right){ }^{180}$ There are rare MOF-derived carbon materials with unprecedented structure-property that necessitate special consideration. An MOF acquired using multi-walled carbon nanotubes (MWCNTs) exhibited an adsorption performance toward CR of $1600 \mathrm{mg} \mathrm{\textrm {g } ^ { - 1 }}$. $^{181}$ Multiple 1D carbon nanorods and 2D graphene nanoribbons were also employed in the adsorption method. The 1D carbon nanorods were derived via the self-sacrificial and morphologypreserved thermal transmutation of MOF-74. With the assistance of the sonochemical method, the thermal activation of robust carbon nanorods resulted in the construction of graphene nanoribbons with two- to six-layer stiffness. The synthetic characteristics of porous carbon materials can be easily modified using heteroatoms, such as $\mathrm{O}, \mathrm{S}$ and $\mathrm{N}$. Porous carbon was doped nitrogen through the carbonization of ZIF-8/urea composites, exhibiting a high adsorption performance due to its Lewis acid-base, electrostatic and hydrogen bonding interactions. ${ }^{182}$
Thermolysis of MOFs can yield nanoscale materials with a metal matrix due to the coordination of the metal ion with organic linkers. ${ }^{183}$ Co-doped hierarchically porous carbon (Co/HPC) was effectively used for the adsorption of MB. ${ }^{184}$ Also, bimetallic compounds such as Zn/Co ZIFs were applied as precursors for the preparation of porous carbon doped by Co. ${ }^{185} \mathrm{Ni}$-doped porous carbon is also known to exhibit a good adsorption performance such as Ni@C and Ni/PC-CNT. ${ }^{186,187}$ In comparison with the traditional methods, MOF materials derived from metal oxides exhibit unique advantages as follows: (1) the simple synthetic procedures can expedite their large-scale use; (2) the morphology, size, and elemental arrangement of the metal oxides form various MOFs; and (3) the porous structure of the resulting MOFs facilitates the adsorption of distinct precursors such as mesoporous silica and molecular sieves. After the calcination of Ce-BTC in a green solvent, $\mathrm{CeO}_{2}$ nanofibers were derived with a good adsorption capacity $\left(86.6 \mathrm{mg} \mathrm{g}^{-1}\right)$. The adsorption performance of the $\mathrm{CeO}_{2}$ nanofibers is due to their $\pi-\pi$ interactions and electrostatic interactions. ${ }^{188}$ On the other hand, NiO resulted in excellent adsorption capability due to the thermolysis of the precursor at $350{ }^{\circ} \mathrm{C} .{ }^{189}$ In comparison with $\mathrm{C}_{3} \mathrm{~N}_{4}$ and $3 \mathrm{D}$ graphene, the resulting hierarchical meso/microporous structural composites displayed extraordinary adsorption ability ${ }^{190}$ (Table 6).

\section{Possible adsorption mechanisms}

The adsorption of deadly pollutants from wastewater using MOFs as adsorbents has attracted significant attention. The adsorption can occur via distinct mechanisms, i.e., interactions between acid and base, electrostatic interactions between adsorbates and adsorbent, H-bonding, hydrophobic interactions and $\pi-\pi$ stacking (Fig. 10). ${ }^{230}$ It is well documented in the literature that more than one interaction is possible during the adsorption process.

\subsection{Electrostatic interactions}

The most often recognized phenomenon is electrostatic interactions throughout the course of the adsorption process for the elimination of hazardous substances from wastewater. The surface charge is the electric charge formed when the MOF adjusts its net surface charge at the interface and upon dispersion in polar media, i.e., water, which depends on the $\mathrm{pH}$ of the medium. Using the protonation and deprotonation process, the net charge of an MOF can also be changed. ${ }^{231,232}$ Thus, the charge (cationic or anionic) of MOFs can comfortably interact with various charged adsorbates, which is known as electric interactions. In 2010, Haque et al., first reported the adsorption of organic pollutants employing MOFs as adsorbents. ${ }^{231}$ In their study, they synthesized two porous Cr-based MOFs, i.e., MIL-101-Cr and MIL-53-Cr, where MIL stands for Material of Institute Lavoisier. These two MOFs efficiently adsorbed the dangerous anionic methyl orange (MO) dye from wastewater, and their capacity was much higher than that of activated carbon. Furthermore, the adsorption performance of MIL-101-Cr 


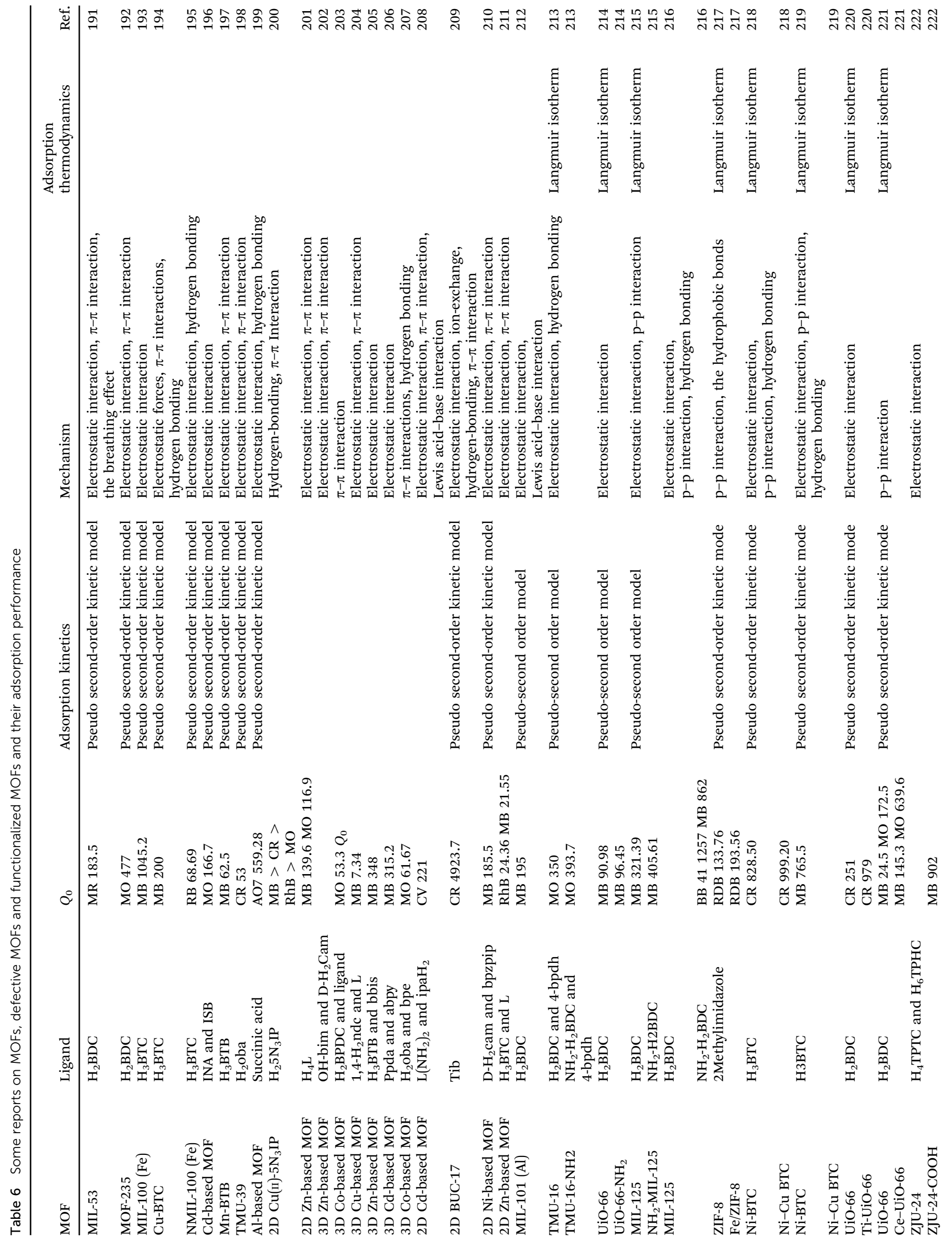


was much better than that of MIL-53-Cr, which additionally supports the importance of the porosity of MOFs in their adsorption characteristic. Also, after the grafting of ED and protonated $\mathrm{ED}$, the adsorption performance of MIL-101-Cr was further enhanced. The anionic dye MO possessing a sulfate ion exhibits electrostatic interaction with cationic MOFs, which are very robust in nature. Using a functionalized MOF, Haque et al., reported the adsorption of methylene blue (MB) and methyl orange (MO) in wastewater. ${ }^{233}$ They attached an amino group on the MOF, and the resulting $\mathrm{NH}_{2}$-MIL-101-Al exhibited a superior adsorption capacity for MB of $762 \pm 12 \mathrm{mg} \mathrm{g}^{-1}$, which is much higher than that of other reported MOFs and materials (Fig. 11).

In the non-functionalized MOF, the adsorption capacity reported for MIL-101-Al is $195 \mathrm{mg} \mathrm{g}^{-1}$, which indicates that the electrostatic interaction is singularly responsible for the high adsorption capacity of $\mathrm{NH}_{2}$-MIL-101-Al. Moreover, Haque et al., fabricated an iron terephthalate-based MOF denoted as MOF-235, which could promptly adsorb $\mathrm{MB}$ and MO dye from wastewater. ${ }^{234}$ Subsequently, the adsorption capability of uranine over MIL-101-Cr was also calculated, which was observed to be $126.9 \mathrm{mg} \mathrm{g}^{-1}$. This high capacity of over MIL-101-Cr is due to the electrostatic interaction between the MOF material and dye molecules. Lin et al., also constructed a Cu-BTC MOF, which showed a good adsorption performance towards MB. ${ }^{235}$ Khushboo et al., reported two coordination polymers based on $\mathrm{Co}(\mathrm{II})$ and $\mathrm{Cu}(\mathrm{II})$ ions, which showed a good adsorption performance towards the cationic dye MB. However, the Co-MOF showed a superior adsorption towards MB dye due to interaction between the cation and aromatic system, i.e. cation $-\pi$ interaction, which is a strong interaction and plays a key role in molecular recognition (Fig. 12). ${ }^{236}$

\subsection{Interactions between acid and base}

The interactions between acids and bases are rarely exist in the adsorption of dangerous pollutants from wastewater. However, the acid-base interaction was observed in the adsorption of an organic pollutant by an MOF. Hasan et al., reported the adsorption of naproxen and clofibric acid on an MIL-101-Crfunctionalized MOF possessing acidic and basic groups. ${ }^{237}$ MIL-101-Cr is an outstanding adsorbent for the elimination of clofibric and naproxen, ${ }^{238}$ but when the acidic $\mathrm{SO}_{3} \mathrm{H}$ and primary $\mathrm{NH}_{2}$ groups were introduced, this increased the acid-base interaction between the MOF and pollutant. Thus, the MOF functionalized with primary $\mathrm{NH}_{2}$ groups exhibited a higher adsorption capacity than that of the pristine MOF, and the opposite was established for the MOFs having $\mathrm{SO}_{3} \mathrm{H}$ groups; therefore, these results are in good agreement with previous reports. ${ }^{239}$

\subsection{Hydrogen bonding}

During the adsorption of hazardous materials, the adsorption mechanism due to hydrogen bonding between the MOF and pollutants seldom occurs. H-bonding is mainly plausible in organic compounds. Liu et al., investigated the aqueous medium adsorption process of $p$-nitrophenol in phenol on MOFs with various architectures and topologies such as MIL-100-Fe, Cr, and $\mathrm{NH}_{2}$-MIL-101-Al. ${ }^{240}$ All the reported MOFs exhibited comparable and poor adsorption capabilities, but $\mathrm{NH}_{2}$-MIL-101-Al showed a 


\section{Electrostaticinteraction}

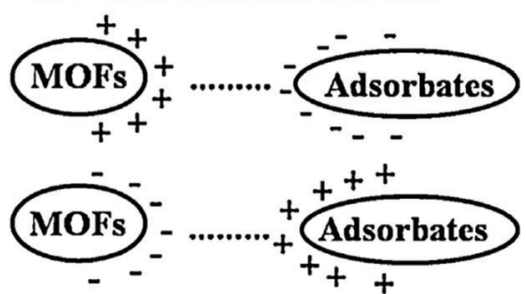

Hydrogen bonding

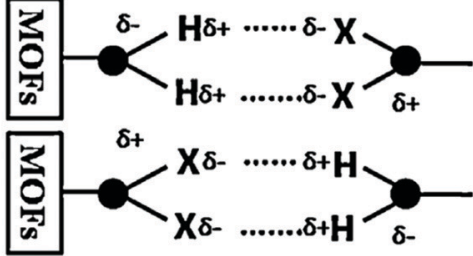

Acid-base interaction

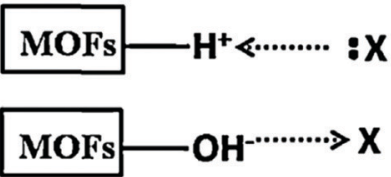

Influence of framework metal

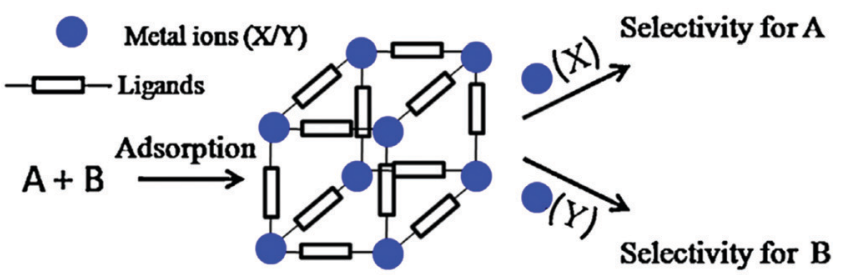

$\pi-\pi$ interaction/stacking

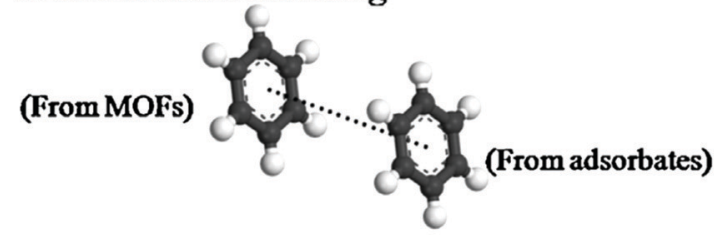

Hydrophobic interaction

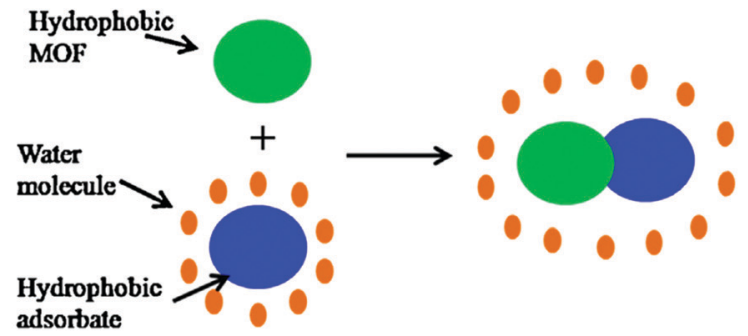

Fig. 10 Schematic diagram of the possible mechanisms for the adsorptive removal of hazardous materials over MOFs. ${ }^{230}$ Reproduced from ref. 230 with permission from Elsevier.

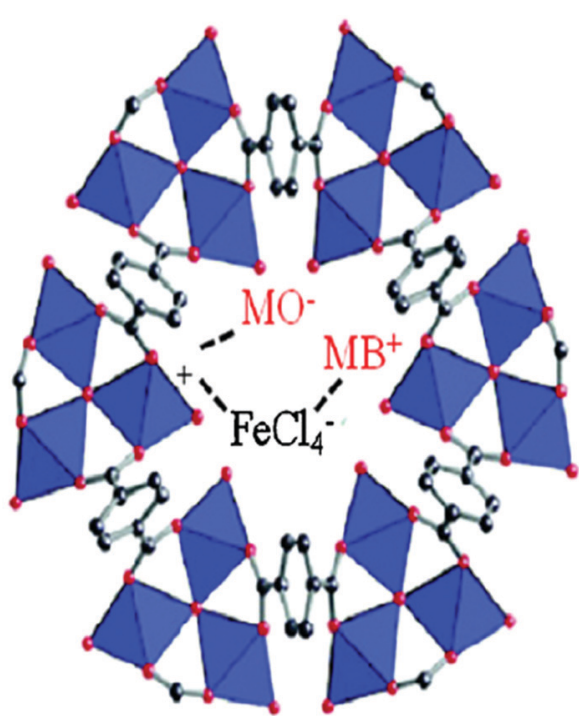

Fig. 11 Electrostatic interaction between $M O$ and $M B$ dyes and the counter positive charge (frameworks) and negative charge (chargebalancing anion) of MOF-235. ${ }^{234}$ Reproduced from ref. 234 with permission from Elsevier.

tremendous adsorption capacity, which was 4.3 and 1.9 times higher than that of MIL-100-Fe and MIL-100-Cr, respectively. The adsorption capability in the case of PNP was much higher for
$\mathrm{NH}_{2}$-MIL-101-Al in phenol than that of AC. This is due to the hydrogen bonding between PNP and the existence of the amino group in $\mathrm{NH}_{2}$-MIL-101-Al (Fig. 13). In an exceptional publication, Xie et al., illustrated the adsorption capacity of two aluminumbased MOFs, CAU-1 and MIL-68-Al, for nitrobenzene from wastewater, where their adsorption capacities were $970 \pm 10$ and $1130 \pm 10 \mathrm{mg} \mathrm{g}^{-1}$, respectively, which are much higher than the experimental values for the porous materials summarized so far. ${ }^{241}$ Due to the presence of -OH groups in Al-O-Al units, both MOFs exhibited a very high adsorption capacity via the creation of $\mathrm{H}$-bonding between the $-\mathrm{OH}$ of the MOF and nitrogen atom of nitrobenzene (Fig. 13).

Subsequently, H-bonding in the adsorption of carbon dioxide and ammonia with MOFs or functionalized MOFs ${ }^{242,243}$ was reported in the literature, even when the adsorption was performed in gaseous media. Recently, Mantasha et al., reported two 2-D MOFs $[\mathrm{M}(\mathrm{ox})(\mathrm{bpy})]_{n}\left[\mathrm{H}_{2} \mathrm{Ox}=\right.$ oxalic acid and bpy $=4,4^{\prime}$-bipyridine] based on $\mathrm{Co}$ (II) and $\mathrm{Cu}$ (II) ions, which showed tremendous selective adsorption towards methylene blue even in the presence of methylene orange dye. The mechanism was proposed to occur via electrostatic, $\pi-\pi$ and H-bonding interactions (Fig. 14). ${ }^{244}$

\section{$4.4 \pi-\pi$ interactions}

$\pi-\pi$ interaction is also responsible for the adsorption of organic pollutants over MOFs, ${ }^{245,246}$ but in wastewater adsorption, $\pi-\pi$ 


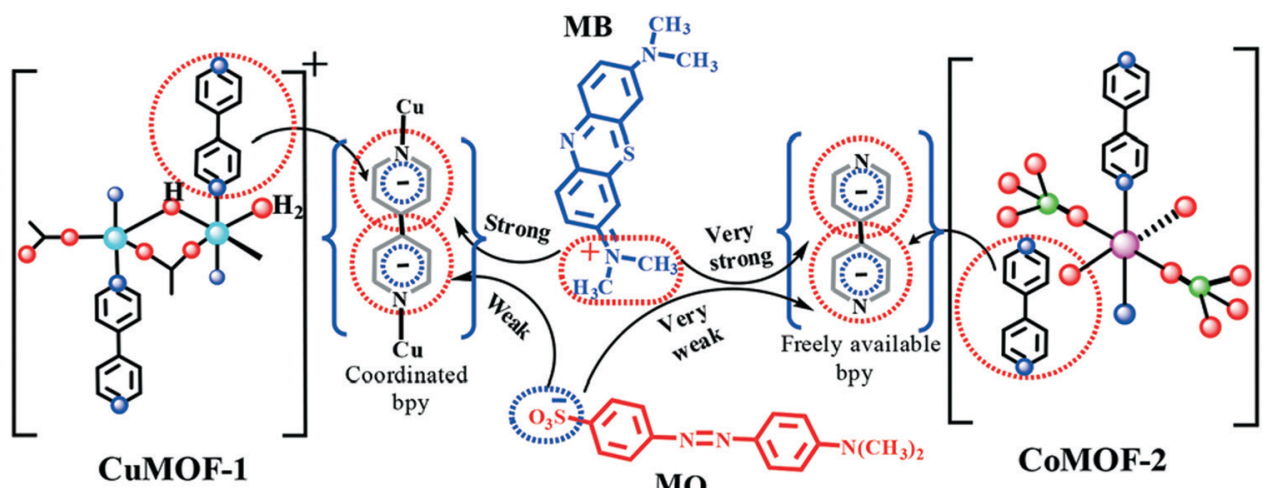

Fig. 12 Plausible mechanism of dye adsorption showing the cation/anion- $\pi$ interaction between cationic/anionic dyes and MOFs. ${ }^{236}$ Reproduced from ref. 236 with permission from the Royal Society of Chemistry.
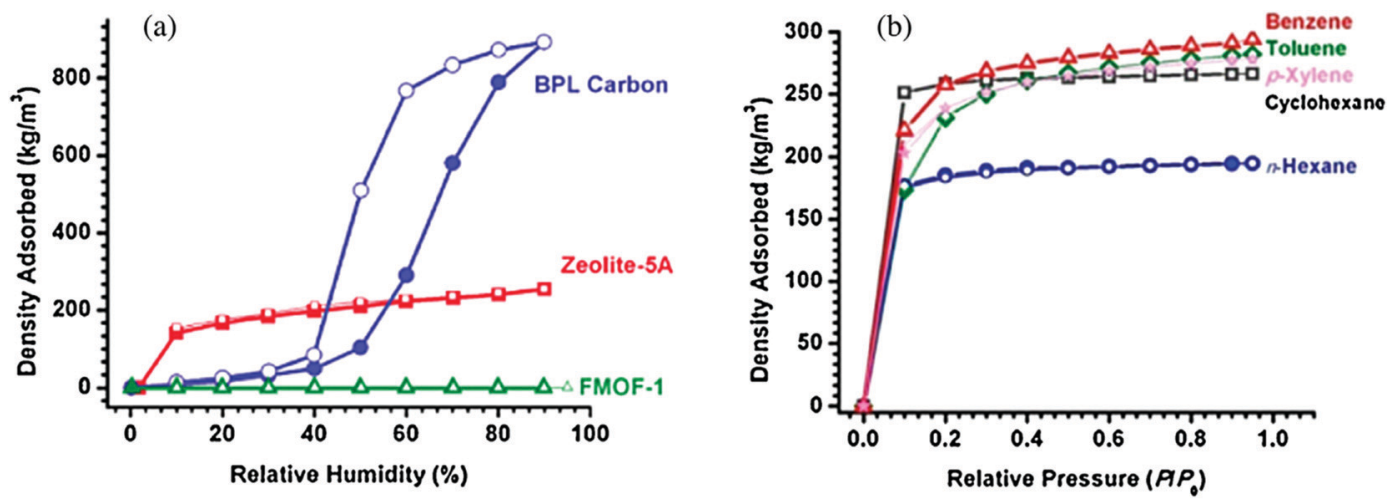

Fig. 13 (a) Water adsorption isotherms over FMOF-1, zeolite-5A, and BPL carbon and (b) adsorption of oil components in FMOF-1 using vapors of cyclohexane, $n$-hexane, benzene, toluene, and $p$-xylene. Open symbols indicate desorption. ${ }^{242}$ Reproduced from ref. 242 with permission from the American Chemical Society.

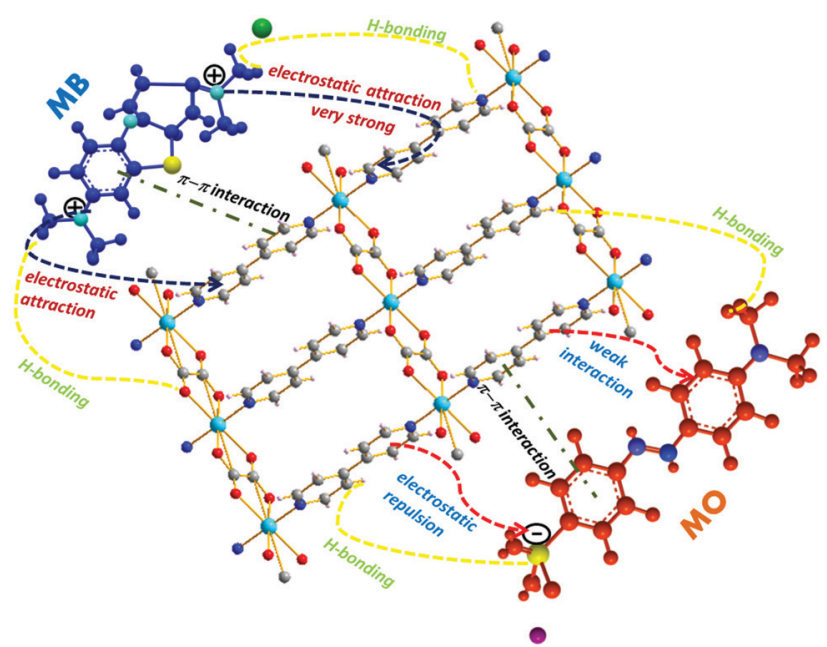

Fig. 14 Plausible mechanism of dye adsorption between cationic/anionic dyes and MOFs (1 or 2). ${ }^{244}$ Reproduced from ref. 244 with permission from Elsevier.

interactions/stacking mainly rather than complexation. Qin et al., reported the removal of bisphenol-A (BPA) using two highly porous MOF materials, MIL-101-Cr and MIL-100-Fe, and the results were analogous to that with AC. ${ }^{247}$ The kinetic parameters obtained in this case followed the order of AC $<$ MIL-100-Fe $<$ MIL-101-Cr. It was speculated that this trend may be attributed to the average pore diameter of the materials. Nevertheless, the adsorption mechanism was not been reported, but the most favorable reason for the high adsorption of BPA over MIL-101-Cr is due to the interactions between the benzene rings of BPA and MIL-101, together with partial H-bonding. Analogous interactions were also proposed in the adsorption of BPA over MIL-5 $3^{248}$ and malachite green over MIL-100-Fe. ${ }^{249}$ Recently, Khalid et al., reported the synthesis of a $\mathrm{Cu}(\mathrm{II})$-based coordination polymer named CP (1), which exhibited a tremendous adsorption performance for cationic and anionic dyes in aqueous media (Fig. 15). ${ }^{250}$ CP (1) adsorbed almost 98\%, 92.8\% and 95\% of $\mathrm{MB}, \mathrm{MO}$ and Rh-B organic dye in only $240 \mathrm{~min}$. The mechanism is attributed to the strong $\pi-\pi$ interactions between the aromatic backbones of the organic dyes with the benzene ring of CP (1). ${ }^{250}$

\subsection{Hydrophobic interactions}

Generally, hydrophobes are non-polar and low soluble molecules with long carbon chains. Hydrophobic interactions are generally observed when adsorption occurs in the aqueous phase. For the adsorption of spilled oil, MOF materials have 
(a)

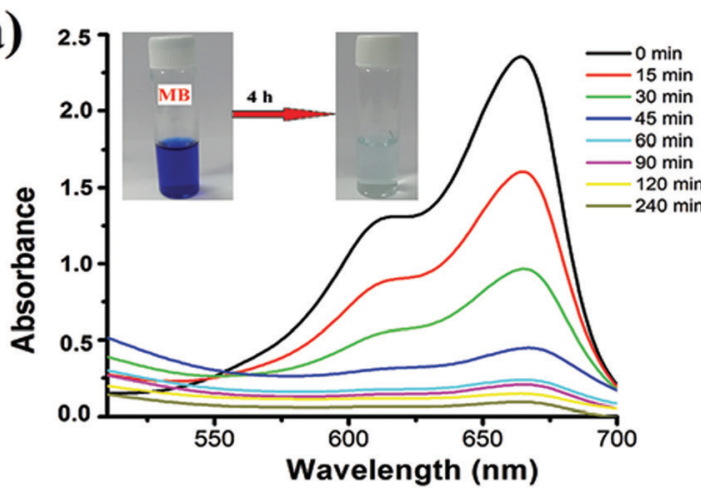

(c)

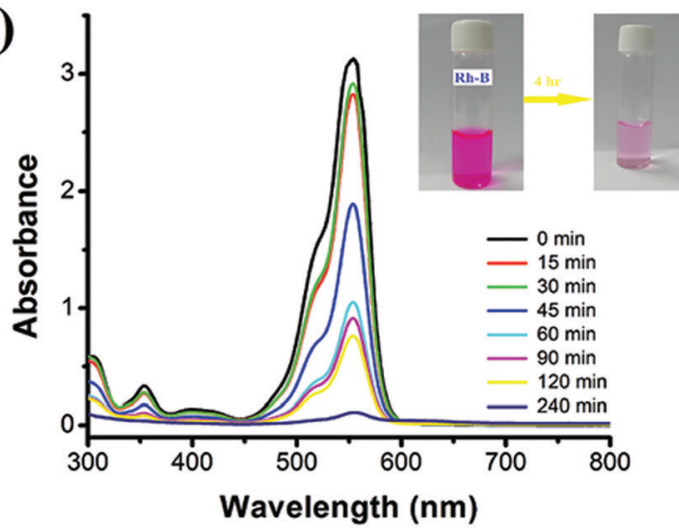

(b)
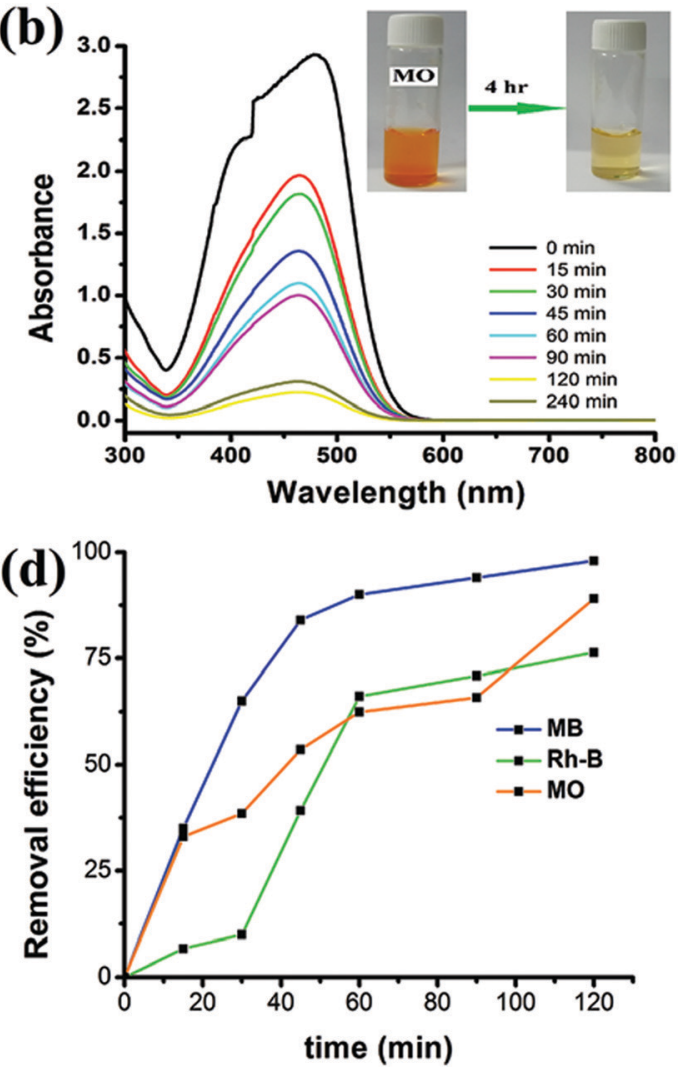

Fig. 15 Change in absorption spectrum of CP (1) in the presence of different dyes: MB (a), MO (b), and Rh-B (c) at different time intervals and (d) efficiency of CP (1) to remove different dyes. ${ }^{250}$ Reproduced from ref. 250 with permission from the Royal Society of Chemistry.

been used as adsorbents. In 2011, Yang et al., reported a fluorous MOF (FMOF-1) design by utilizing silver(I) 3,5-bis((trifluoromethyl)-1,2,4-triazolate) for the adsorption of oil spills. ${ }^{251}$ Because of its perfluorinated inner surface, FMOF-1 is a hydrophobic environment, and thus it exhibited high capacity for C6-C8 hydrocarbons although it has no noticeable hydrocarbons. Lin et al., reported the high extraction of oil droplets (soybean oil) from water over Cu-BTC. ${ }^{252}$ This high adsorption is attributed to the hydrophobic interactions between soybean oil and the benzene rings of the Cu-BTC MOF.

\subsection{Photocatalytic degradation of dyes}

Besides adsorption, sometimes organic pollutant dyes can be removed from wastewater through degradation via the mechanism of oxidation by specially employing photocatalysts. ${ }^{253,254}$ The degradation of organic pollutants via the oxidation method, which includes ozonation, Fenton reaction, and photocatalysis, is applied due to its high efficiency, better recyclability, low coast, and safe handling. ${ }^{254-259}$ The advance oxidation process is more advantageous than other processes because it transforms of organic pollutants into less harmful ions. Photocatalysis for the treatment of wastewater via the advance oxidation process involves the use of semiconductors such as $\mathrm{TiO}_{2}, \mathrm{ZnS}, \mathrm{GaP}, \mathrm{ZnO}$, and $\mathrm{Fe}_{2} \mathrm{O}_{3}$ as catalysts to effectively degrade organic dyes into less toxic ions. In most cases, the photocatalyst even mineralizes these less toxic molecules into $\mathrm{H}_{2} \mathrm{O}$ and $\mathrm{CO}_{2} \cdot{ }^{259-262}$ The advantages of photocatalytic reactions are as follows: (a) these reactions occur at ambient temperature and pressure; (b) they almost mineralize the initial and intermediate compounds without producing other toxic pollutants and (c) they are inexpensive to perform. ${ }^{253,254}$ However, the disadvantage of this method is that the semiconductors employed as catalysts are not very photostable, resulting in the corrosion of the catalyst in aqueous media, and further the movement of metal ions into water and dissolution of the solid catalysts. To date, many semiconductors have been explored for the photocatalytic degradation of organic pollutants, but $\mathrm{TiO}_{2}$ remains the best one due to its low toxicity, exceptional stability, and economical cost. ${ }^{263,264}$ However, it also involves some drawbacks such as low photocurrent quantum yield and reduced solar energy utilization efficiency. Henceforth, the development of new materials as photocatalysts with better degradation performances and stability is crucial. ${ }^{253,254}$ Recently, MOF materials have been reported as a new class of photocatalysts for the degradation of organic dyes under UV and visible irradiation. Due to their abundant metalcontaining nodes, various organic linkers and controllable synthetic process, it is effortless to design MOFs with better degradation capableness and tailored dimensions to absorb light. Consequently, the photocatalytic application of MOFs has an promising future, although they have not been exploited much 
compared to traditional metal oxides and sulfides. MOF-5 was the first reported MOF to function as a photocatalyst, ${ }^{265}$ which was composed of clusters of $\mathrm{Zn}_{4} \mathrm{O}$ situated at the edge of the framework, joined by 1,4-bdc organic ligands.

The photodegradation mechanism is directly related to the band gap energy and other structural features supporting the degradation process. The MOF exhibited broadband adsorption in the range of $500-800 \mathrm{~nm}$, which is attributed to the delocalized electron existing on the microsecond time scale remaining on its conduction band (CB). MOF-5 resulted in the enhanced degradation of phenol in wastewater comparable to that of $\mathrm{TiO}_{2}$ semiconductors. Encouraged by the photocatalytic activity of MOF-5, Chen et al., designed a porous MOF, $\left[\mathrm{Zn}_{4} \mathrm{O}(2,6 \mathrm{ndc})_{3}(\mathrm{DMF})_{1.5}\left(\mathrm{H}_{2} \mathrm{O}\right)_{0.5}\right] \cdot 4 \mathrm{DMF} \cdot 7.5 \mathrm{H}_{2} \mathrm{O}$, which was named UTSA-38, having a bandgap energy of $2.85 \mathrm{eV}$ for the photodegradation of MO in aqueous phase. ${ }^{266}$ It was established that this appropriate MOF degraded the MO dye in the visible region. However, under UV light, the degradation of MO occurred rapidly within only $120 \mathrm{~min}$, suggesting that UV light may more effectually degrade organic dyes than visible light (Fig. 16). ${ }^{255}$ The plausible mechanism for the degradation of the organic dye by the MOF is shown in Fig. 16, which indicates that initially electron-hole pairs are formed in UTSA-38. The subsequent absorption of light and the promotion of the electrons from the valence band $(\mathrm{VB})$ to the conduction band $(\mathrm{CB})$ result in the formation of holes in the VB. The holes and electrons move to the surface of UTSA-38 and the electrons reduce oxygen $\left(\mathrm{O}_{2}\right)$ to its radical $\left({ }^{\bullet} \mathrm{O}_{2}\right)$, which is further transformed into hydroxyl radicals $\left({ }^{\bullet} \mathrm{OH}\right)$, and in turn, the holes oxidize the hydroxyl $\left(\mathrm{H}_{2} \mathrm{O}\right)$ to hydroxyl radicals $\left({ }^{\bullet} \mathrm{OH}\right)$. Hydroxyl radicals $\left({ }^{\bullet} \mathrm{OH}\right)$ were proven to be responsible for the decomposition of MO efficiently. ${ }^{267}$

Recently, Cui et al., designed six coordination polymers by employing Ni(II) and $\mathrm{Co}$ (II) ions. The photocatalytic degradation capacity of complexes 1-6 for methylene blue (MB) was investigated, and surprisingly complexes 1-4 exhibited a better photocatalytic degradation effect for MB (Fig. 17). ${ }^{268}$ The degradation follows the first order rate equation and complex 6 showed an enhanced degradation property with an increase in the $\mathrm{pH}$ of the reaction. ${ }^{268}$ Kaur et al., reported a Cd(II)-based metal-organic framework (MOF) formulated as $\left\{\left[\operatorname{Cd}(\mathrm{PA})\left(4,4^{\prime}\right.\right.\right.$ bpy $\left.\left.)_{2}\right]\left(\mathrm{H}_{2} \mathrm{O}\right)\right\}_{n}$ employing pamoic acid and bipyridine ligands. This photocatalyst reduced organic dyes, such as methyl orange (MO) and rhodamine B (Rh-B). Moreover, a Cd(II)-based MOF under visible light irradiation was also explored in the literature [q]. It can be seen in Fig. 18 that the Cd(II)-based MOF showed a better degradation property in the presence of $\mathrm{H}_{2} \mathrm{O}_{2}$. Moreover, the kinetics of the reaction was also investigated, which suggests a pseudo-first-order process for the degradation

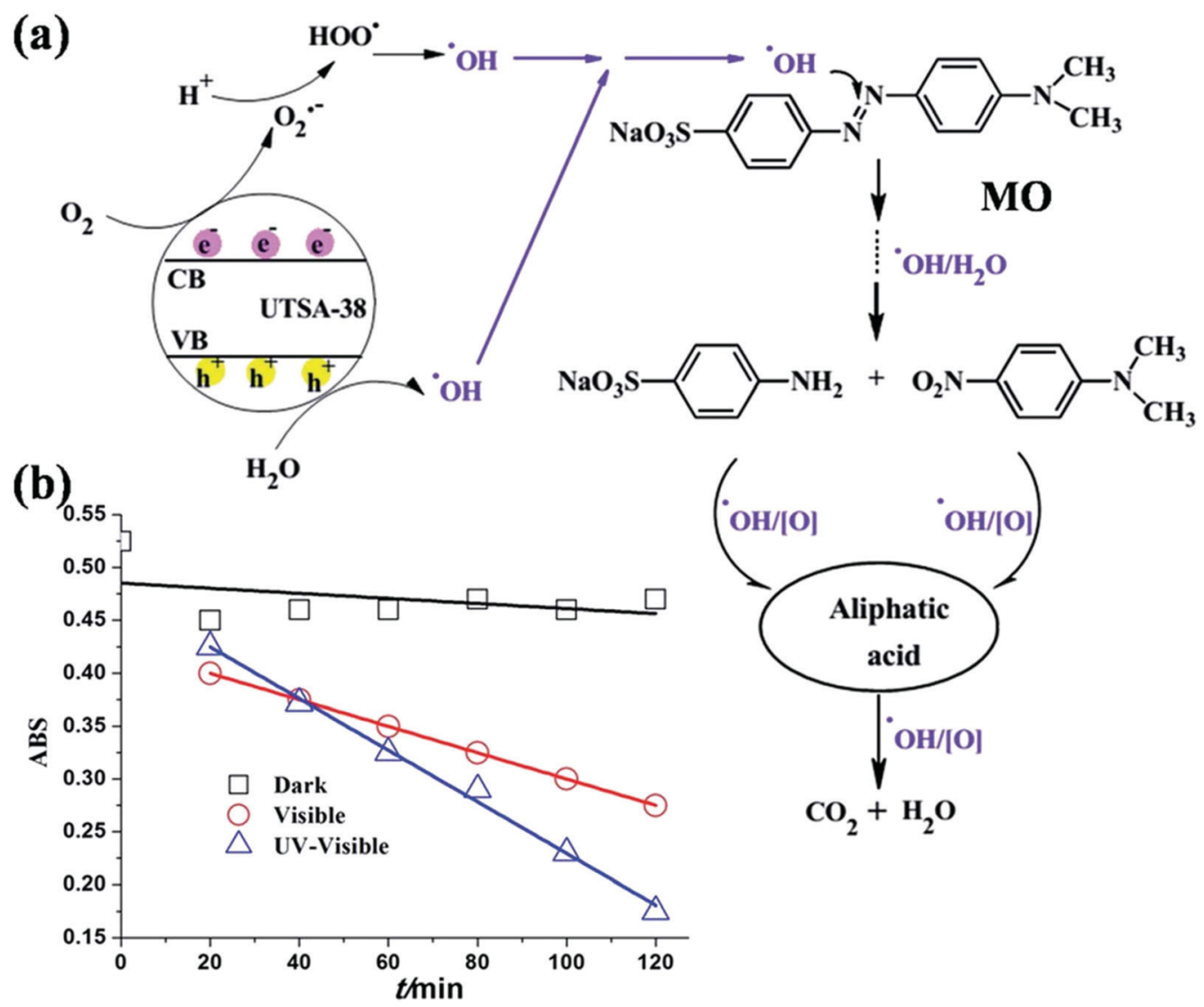

Fig. 16 (a) Main pathways proposed for the photodegradation of methyl orange by UTSA-38 under UV-visible or visible light irradiation. (b) Plots of absorbance of methyl orange solution degraded by UTSA-38 as a function of irradiation time under UV-visible light, visible light and dark. Reproduced from ref. 255 with permission from the Royal Society of Chemistry. 

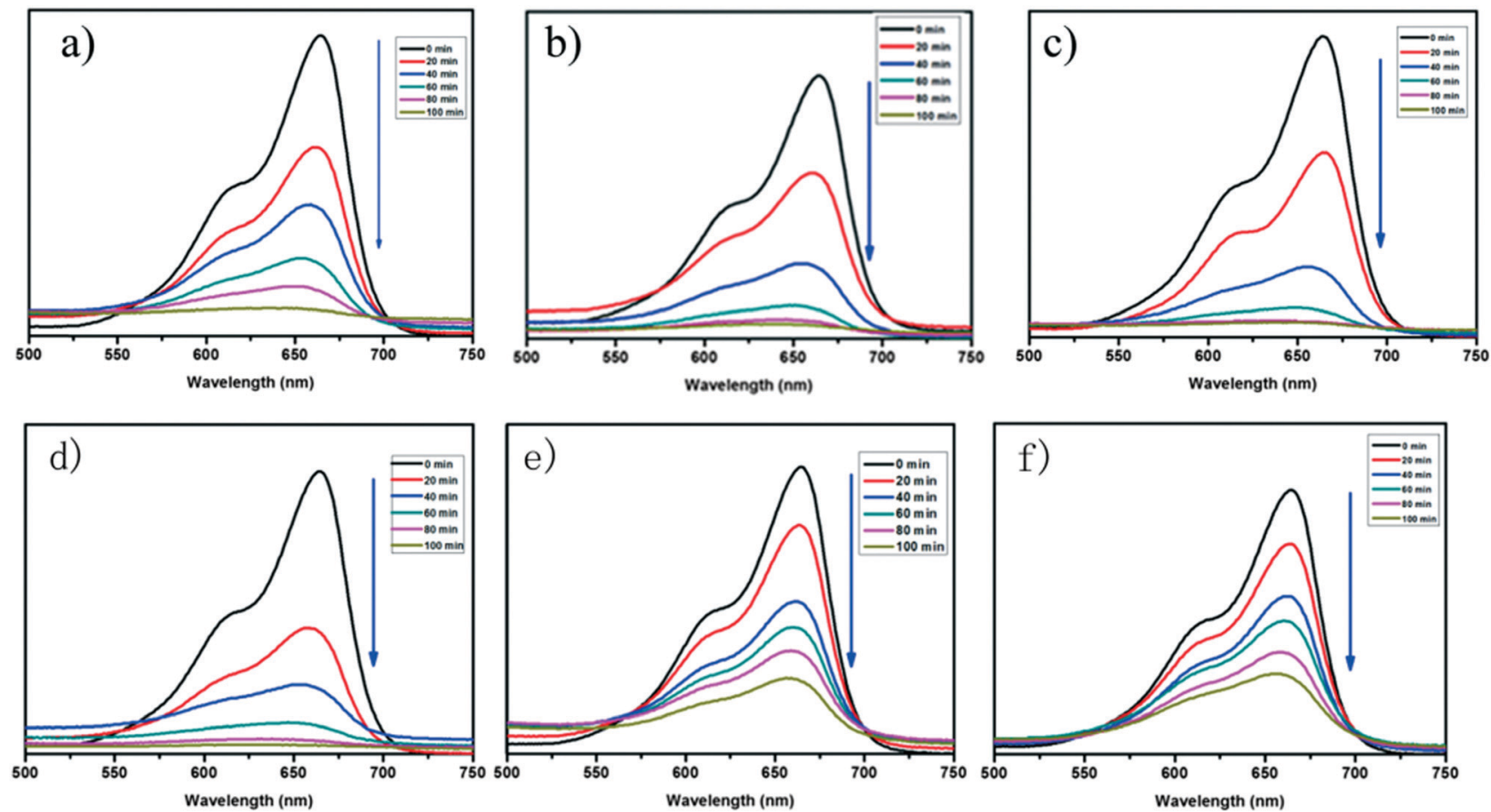

Fig. 17 (a)-(f) Absorption spectra of MB dye solution in the presence of complexes 1-6. Reproduced from ref. 268 with permission from the Royal Society of Chemistry.

of $\mathrm{RhB}$ and $\mathrm{MO}^{269}$ Furthermore, two designed porous materials, $x$ and $y$, also showed better degradation of methylene blue in the UV region. Fig. 19 shows the control experiments under different reaction conditions to reduce the $\mathrm{Cr}(\mathrm{VI})$ to $\mathrm{Cr}(\mathrm{III})$, confirming that the $\mathrm{pH}$ value was indispensable for the reduction of $\mathrm{Cr}(\mathrm{vI}){ }^{270}$ Henceforth, together with dye adsorption, the degradation of dyes also results in the overall removal of pollutants from waste, involving the mechanism of electron-hole availability and the band gap of the CB and VB in MOFs. Thus, by suitable designing MOFs, hazardous aromatic dyes can be adsorbed (by degradation), controlling the mechanism of photocatalysis.

\subsection{Role of the theoretical approach (DFT) in the host-guest chemistry of MOFs}

Density functional theory together with other theoretical tools such as molecular dynamic (MD) simulations have become an intriguing part in the study of MOFs for functional applications. The dynamic and kinetic aspects of substrate binding within porous MOFs are important to understand the host-guest interaction properly. ${ }^{271}$ In particular, theoretical chemists have focused on studying host-guest interactions, which consider the open metal sites or pendant functional groups in the pore as the primary binding sites for incoming guest entities. In the field of gas storage, separation and purification, special emphasis has been placed on the interactions between MOF hosts and the adsorbed substrate molecules, leading to the discovery of new functional materials with higher storage capacities and stronger binding energies. Owing to their importance, the investigation of the guest-host binding interactions involved in the adsorption becomes significant to determine how these materials (MOFs) function, and to elaborate the mechanisms for their sensing and discrimination of guest molecules such as dyes.

Computational modelling, including density functional theory (DFT) and molecular dynamics (MD), linked to experimental data has played an important role in visualizing the molecular motions and diffusion of guest molecules within the pores of MOFs. The combination of these static, dynamic and kinetic approaches offers a comprehensive understanding of the guest-host binding processes, which ultimately govern the properties of MOFs. The nature of the host-guest interactions is often related to weak supramolecular mechanisms (e.g., hydrogen bonds, $\pi-\pi$ interactions, van der Waals, electrostatic and dipole interactions). These supramolecular interactions often involve hydrogen atoms and undergo dynamic processes. The dynamics of these interactions are difficult to probe directly by conventional spectroscopic experiments such as infrared (IR) and Raman spectroscopy, which are the most common methods to study molecular vibrations. However, it is easy to understand these interactions using DFT analysis.

Considering dyes as guest molecules, exact theoretical insights into their adsorption have not been established; however, the mechanism for the degradation of dyes has been detailed by some theoretical chemists, for example Abhinav et al. This group established a plausible mechanistic pathway for the photodegradation of aromatic dyes in the presence of an MOF by performing band structure calculations for the MOF under investigation. This calculation was based on the DFT method. ${ }^{272}$ For example, they demonstrated the density of states (DOS) and partial density of states (pDOS) for the MOF, as shown in Fig. 20. The pDOS plots show that the valence band 
(a)
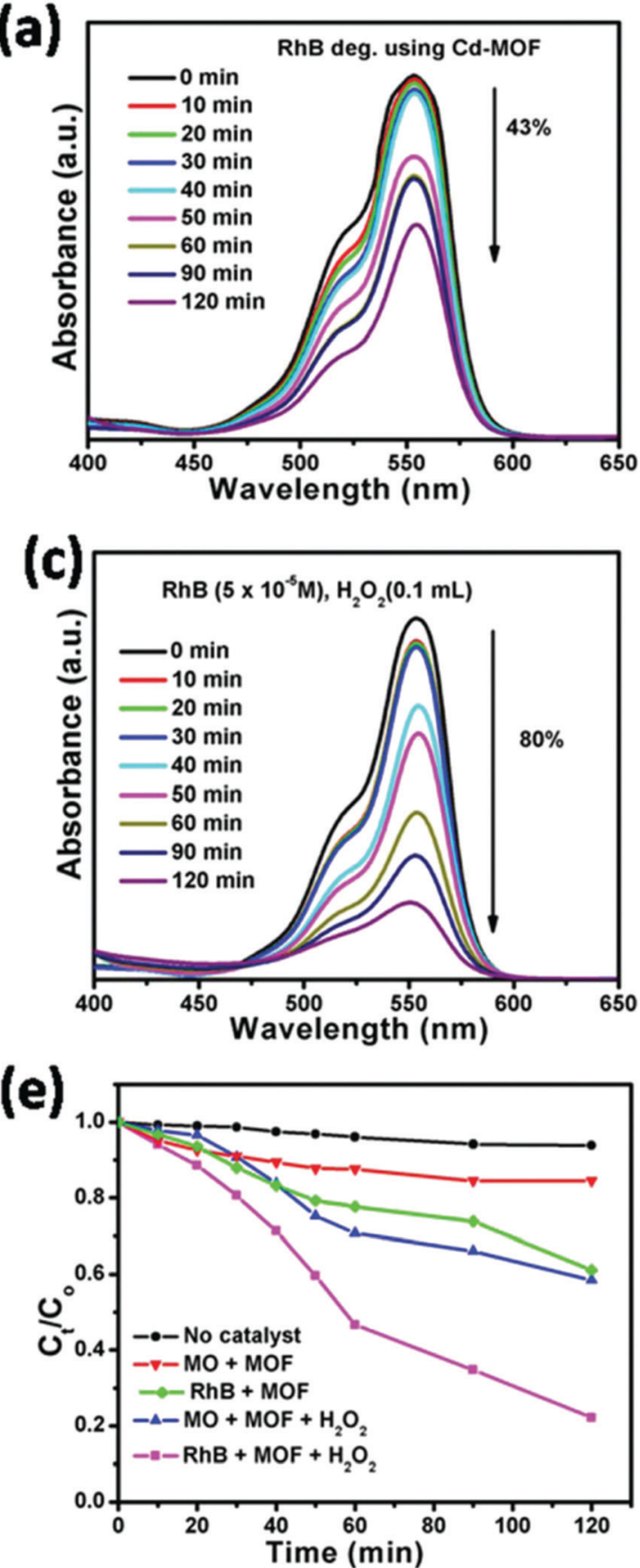

(b)

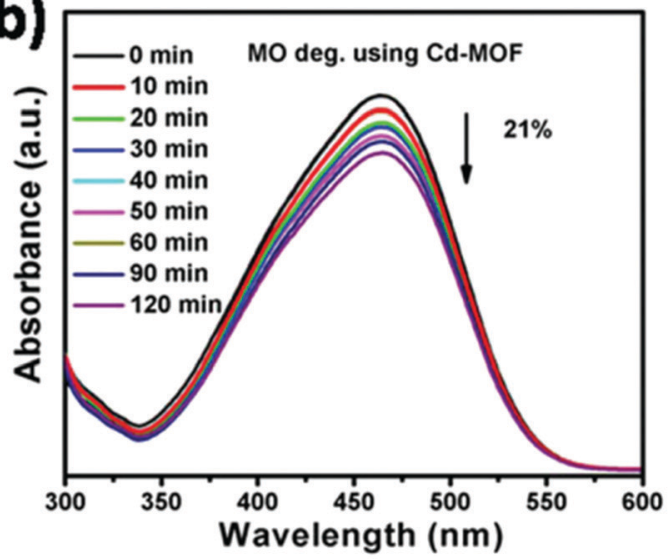

(d)

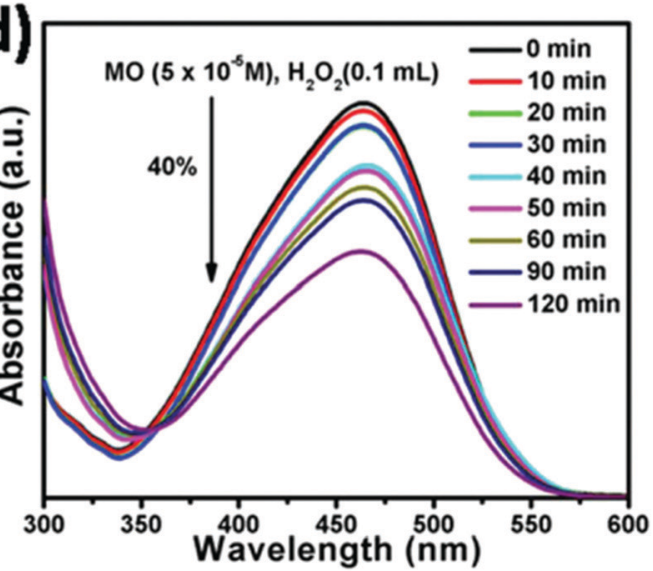

$(\mathbf{f})$

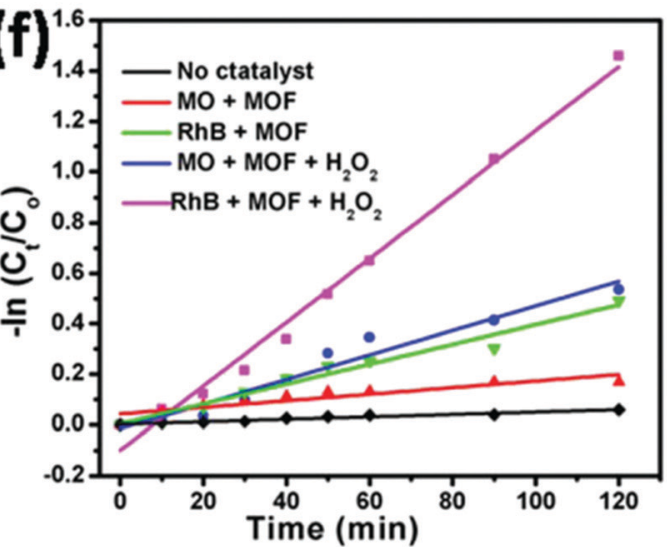

Fig. 18 UV-vis spectra for the photocatalytic degradation of RhB and $\mathrm{MO}(\mathrm{a})$ and (b) over Cd-MOF and (c) and (d) with the addition of $\mathrm{H}_{2} \mathrm{O}_{2}$. Kinetic plots for the photocatalytic degradation of RhB and MO under various parameters (e) and (f). Reproduced from ref. 269 with permission from the Royal Society of Chemistry.

in the MOF originates mainly from the aromatic carbon centers and oxygen centers of the carboxylate groups with a small contribution from the zinc centers. In addition, the conduction band in the range of -0.35 to $0.87 \mathrm{eV}$ is attributed to the carbon centers with an additional contribution by the oxygen centers. Therefore, DOS and pDOS plots disclosed that the electronic transitions in the MOF occur from the aromatic to aromatic center (ligand-to-ligand) with an additional contribution from the carboxylate oxygen centers. Referring to this, it is established (in this case) that during photo-excitation, charge transfer occurs from the HOMO to the LUMO to generate holes, and the HOMO strongly needs one electron to return to its stable state. Thus, one electron taken from a water molecule is converted to the ${ }^{\bullet} \mathrm{OH}$ active species, which decompose the dye effectively to complete the photocatalytic process. ${ }^{272}$

The theoretical studies described herein for the elucidation of the binding sites, host-guest interactions and photodegradation of dyes with MOFs can enable and assist the 

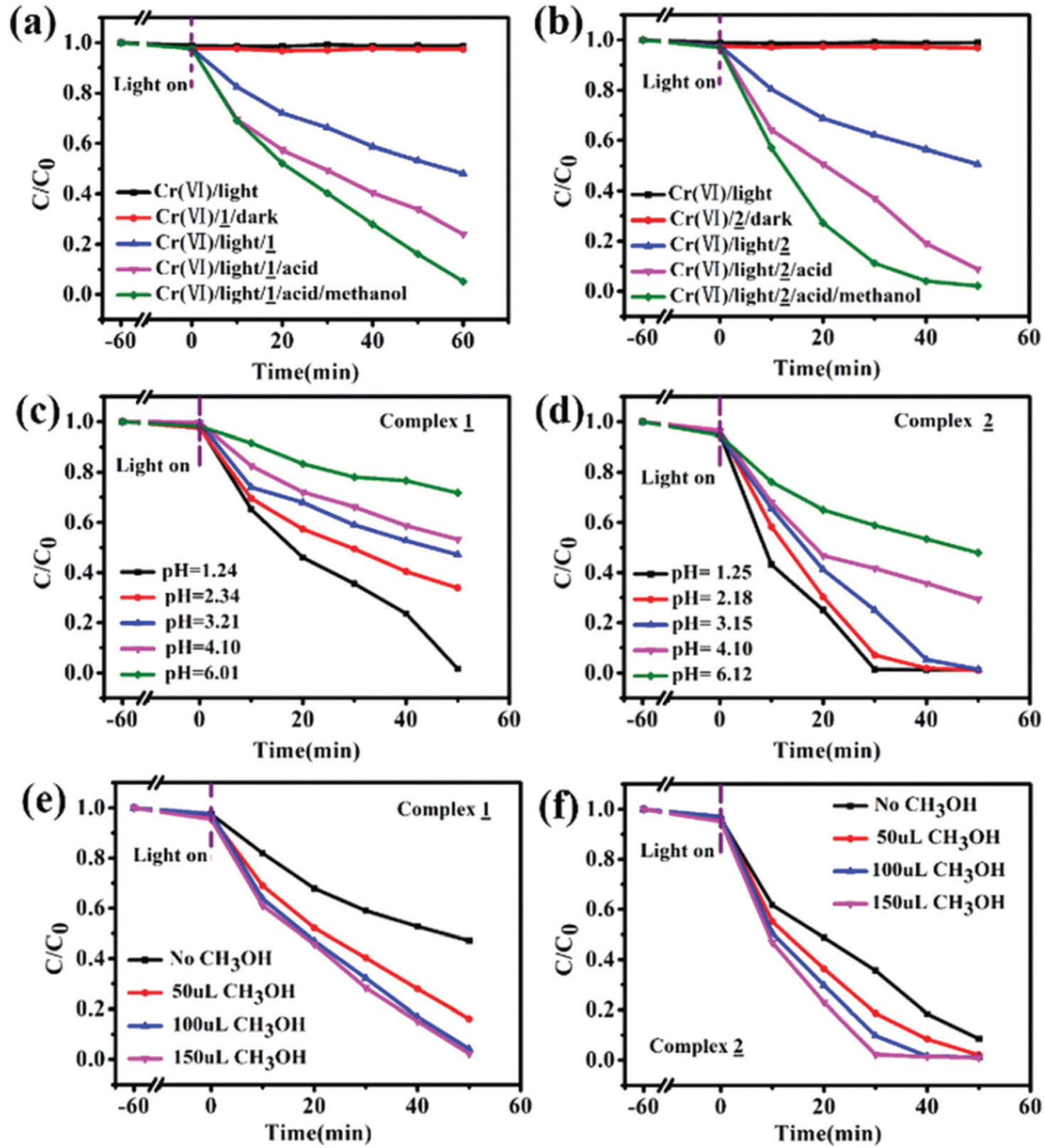

Fig. 19 Control experiments for the reduction of $\mathrm{Cr}(\mathrm{vI})$ over complexes 1 (a) and 2 (b) under different conditions; photocatalytic reduction of $\mathrm{Cr}(\mathrm{vI})$ over complexes 1 (c) and 2 (d) at different pH values; and photocatalytic reduction of $\mathrm{Cr}(\mathrm{VI})$ complexes 1 (e) and 2 (f) with different methanol addition. Reproduced from ref. 270 with permission from the Royal Society of Chemistry.

design and optimization of functional materials, in which high adsorption capacity, selectivity and stability may be achieved simultaneously.

\section{Conclusions and future prospects}

In summary, we focused the current perspectives of the properties of MOFs and their dye adsorption capacities together with the factors and mechanisms influencing their material properties. Metal-organic frameworks (MOFs) are a different class of strong materials having a highly expanded structure and are probable candidates as adsorbents for the removal of organic pollutants from wastewater. Currently, there are various review articles in the literature on the removal of organic dyes from wastewater using MOF materials. These review articles indicate the strong interest shown by the research community in the treatment of wastewater using MOFs as adsorbents. MOFs show improved and enhance adsorption capacity and kinetics in comparison to that of the conventional used adsorbents such as AC and zeolites due to their easier tunability than that of conventional methods. Due to their large surface area, tunable porosity and ability for functional integration, MOFs are high quality materials compared with conventional adsorbents. The adsorption of organic dyes on 


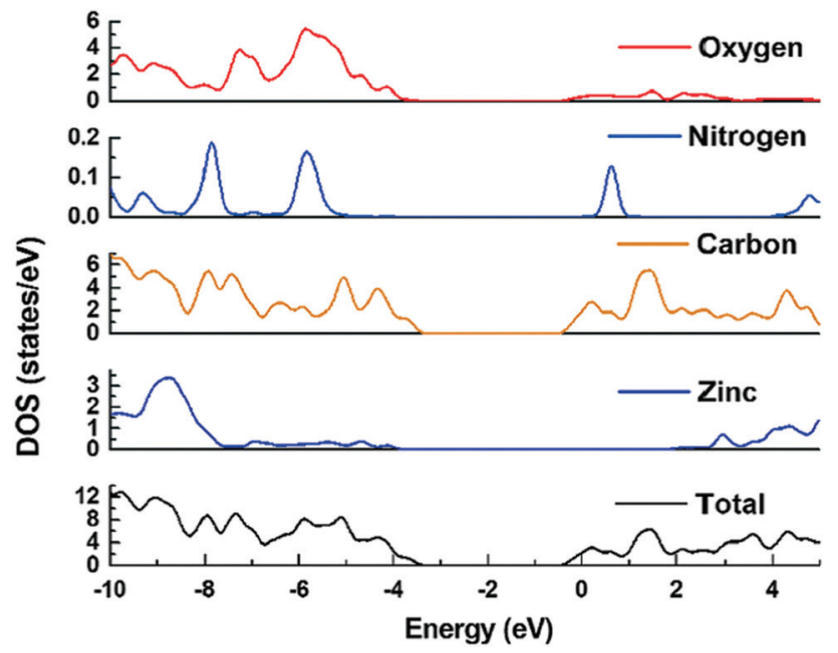

Fig. 20 DOS and partial DOS plots (obtained from DFT) for Zn-MOF. Reproduced from ref. 272 with permission from the Royal Society of Chemistry.

MOFs is due to various electrostatic interactions, hydrogen bonding, $\pi-\pi$ interactions and acid-base interactions between the adsorbent and adsorbates. The adsorption mechanism mainly follows a specific interaction depending on the target dye and structure or chemistry of the MOF. MOFs can be easily tuned for their functional application via the specific selection of the organic linker in the synthetic and post-synthetic process. In addition, the methodology for the synthesis of MOFs influences their surface area, size, shape, expansion of pores, chemical environment and dimensions. Besides, the advantages and the critical factors to employ MOF-based materials as dye adsorbents including their high efficiency, water stability, simple operation process, cost effectiveness, less amount of hazardous side products, they also suffer from some drawbacks, which need to be overcome. These drawbacks include their inability to adsorb certain dyes (either owing to the complex structures of the dye molecules or the absence of appropriate porous sites to interact with the dye moiety), their costly regeneration, disposal of the adsorbent residue, and loss of adsorbent. These issues need to be addressed to make MOFs more suitable for use in effective dye adsorption in future endeavors. Thus, it can be concluded that advance research is necessary to understand the interactions between MOFs and organic pollutants to enhance the properties of MOFs and apply them as adsorbents for the removal of organic dyes from wastewater. In addition, we hope that the scientific community will focus on the various technologies for designing water-stable MOFs with the aim of incorporating water resistance into MOFs.

\section{Conflicts of interest}

There are no conflicts to declare.

\section{References}

1 D. Seckler, R. Barker and U. Amarasinghe, Int. J. Water Resour. D, 1999, 15, 29-42.
2 S. Malato, P. Fernandez-Ibanez, M. I. Maldonado, J. Blanco and W. Gernjak, Catal. Today, 2009, 147, 1-59.

3 M. Rafatullah, O. Sulaiman, R. Hashim and A. Ahmad, J. Hazard. Mater., 2010, 177, 70-80.

4 M. Marta, R. Bruno, J. S. Ferrando-Soria, D. Armentano and E. Pardo, J. Mater. Chem. A, 2018, 6(12), 4912-4947.

5 S. Casani, M. Rouhany and S. Knochel, Water Res., 2005, 39(6), 1134-11346.

6 V. Lazarova, B. Levine and P. Renaud, Wastewater reclamation in Africa: assessment of the reuse applications and available technologies. Proc IXème Congrès de l'Union Africaine des Distributeurs d'Eau; 1998 16-20 Feb; Casablanca.

7 V. Sivakumar and P. G. Rao, Ultrason. Sonochem., 2003, 85-94.

8 J. S. Gajda, H. S. Freeman and A. Reife, Dyes Pigm., 1996, 30, 1-20.

9 G. Zeng, M. Chen and Z. Zeng, Science, 2013, 340, 1403.

10 S.-F. Yang, C.-G. Niu, D.-W. Huang, H. Zhang, C. Liang and G.-M. Zeng, Environ. Sci.: Nano, 2017, 4, 585-595.

11 J.-H. Deng, X.-R. Zhang, G.-M. Zeng, J.-L. Gong, Q.-Y. Niu and J. Liang, Chem. Eng. J., 2013, 226, 189-200.

12 X. Ren, G. Zeng, L. Tang, J. Wang, J. Wan, Y. Liu, J. Yu, H. Yi, S. Ye and R. Deng, Sci. Total Environ, 2018, 610, 1154-1163.

13 M. N. Chong, B. Jin, C. W. K. Chow and C. Saint, Water Res., 2010, 44(10), 2997-3027.

14 I. Ali, M. Asim and T. A. Khan, J. Environ. Manage., 2012, 113, 170-183.

15 M. Yang, J. Environ. Sci. Health, Part C: Environ. Carcinog. Ecotoxicol. Rev., 2011, 29(3), 223-249.

16 S. B. Wang and Y. L. Peng, Chem. Eng. J., 2010, 156, 11-24. 17 I. Ali, Chem. Rev., 2012, 112, 5073-5091.

18 M. S. Khan, M. Khalid, M. S. Ahmad, M. Shahid and M. Ahmad, Res. Chem. Intermed., 2020, 46, 2985-3006.

19 M. Saleem, T. Pirzada and R. Qadeer, Colloids Surf., A, 2007, 292, 246-250.

20 F. Deniz and R. A. Kepekci, J. Mol. Liq., 2016, 219, 194.

21 V. K. Gupta, P. J. M. Carrott, M. M. L. Ribeiro Carrott and Suhas, Crit. Rev. Environ. Sci. Technol., 2009, 39, 783.

22 T. Ngulube, J. R. Gumbo, V. Masindi and A. Maity, J. Environ. Manage, 2017, 191, 35-57.

23 M. A. Jamal, M. Muneer and M. Iqbal, Chem. Int., 2015, 1, 2-6.

24 M. Ismail, W. Zheng, L. Zhang, J. Ma, Y. Jia, Y. Hu and Y. Wang, Chemosphere, 2019, 228, 212-218.

25 Y. Zhou, X. Liu, Y. Xiang, P. Wang, J. Zhang, F. Zhang and L. Tang, Bioresour. Technol., 2017, 245, 266-273.

26 U. Shamraiz, R. A. Hussain, A. Badshah, B. Raza and S. Saba, J. Photochem. Photobiol., B, 2016, 159, 33-41.

27 X. Jiang, J. Shen, S. Lou, Y. Mu, N. Wang, W. Han and L. Wang, Bioresour. Technol., 2016, 216, 645-652.

28 G. Crini, Bioresour. Technol., 2006, 97, 1061-1085.

29 J. R. Long and O. M. Yaghi, Chem. Soc. Rev., 2009, 38, 1201-1507.

30 M. S. Khan, M. Khalid, M. S. Ahmad, M. Shahid and M. Ahmad, J. Struct. Chem., 2019, 60, 1833-1841. 
31 S. Dhaka, R. Kumar, A. Deep, M. B. Kurade, S. W. Ji and B. H. Jeon, Coord. Chem. Rev., 2019, 380, 330-352.

32 J. R. Li, J. Sculley and H. C. Zhou, Chem. Rev., 2012, 112(2), 869-932.

33 Z. Hasan and S. H. Jhung, J. Hazard. Mater., 2015, 283, 329-339.

34 M. P. Suh, H. J. Park, T. K. Prasad and D. W. Lim, Chem. Rev., 2012, 112(2), 782-835.

35 A. R. Millward and O. M. Yaghi, J. Am. Chem. Soc., 2005, 127(51), 17998-17999.

36 J. R. Li, Y. G. Ma, M. C. McCarthy, J. Sculley, J. M. Yu and H. K. Jeong, Coord. Chem. Rev., 2011, 255, 1791-1823.

37 K. Sumida, D. L. Rogow, J. A. Mason, T. M. McDonald, E. D. Bloch and Z. R. Herm, Chem. Rev., 2012, 112(2), 724-781.

38 J. E. Mondloch, M. J. Katz, W. C. Isley, P. Ghosh, P. Liao and W. Bury, Nat. Mater., 2015, 14, 512-516.

39 M. A. Nasalevich, M. van der Veen, F. Kapteijn and J. Gascon, CrystEngComm, 2014, 16(23), 4919-4926.

40 M. S. Khan, M. Khalid, M. S. Ahmad, M. Ahmad, M. Ashafaq, Rahisuddin, R. Arif and M. Shahid, J. Mol. Struct., 2019, 1175, 889.

41 K. K. Wang, H. L. Huang, W. J. Xue, D. H. Liu, X. D. Zhao and Y. Xiao, CrystEngComm, 2015, 17, 3586-3590.

42 H. Furukawa, K. E. Cordova, M. O'Keeffe and O. M. Yaghi, Science, 2013, 341, 1230444.

43 O. M. Yaghi, G. Li and H. Li, Nature, 1995, 378, 703-706.

44 K. A. Cyshosz, A. G. Wong-Foy and A. J. Matzger, J. Am. Chem. Soc., 2008, 130, 6938-6939.

45 S. Kitagawa, R. Kitaura and S. Noro, Angew. Chem. Int. Ed., 2004, 43, 2334-2375.

46 S. Ma and H.-C. Zhou, J. Am. Chem. Soc., 2006, 128, 11734-11735.

47 Y. Liu, V. Ch Kravtsov, R. Larsen and M. Eddaoudi, Chem. Commun., 2006, 8-1490.

48 J. Kim, N. Rosi, D. Vodak, J. Wachter, M. O'Keeffe and O. M. Yaghi, Science, 2002, 295, 469-472.

49 S. Chaemchuen, N. A. Kabir, K. Zhou and F. Verpoort, Chem. Soc. Rev., 2013, 42, 9304-9332.

50 J. Juan-Alcaniz, R. Gielisse, A. B. Lago, E. V. Ramos-Fernandez, P. Serra-Crespo, T. Devic, N. Guillou, C. Serre, F. Kapteijn and J. Gascon, Catal. Sci. Technol., 2013, 3, 2311-2318.

51 Y. K. Hwang, D.-Y. Hong, J.-S. Chang, S. H. Jhung, Y.-K. Seo, J. Kim, A. Vimont, M. Daturi, C. Serre and G. Férey, Angew. Chem. Int. Ed., 2008, 47, 4144-4148.

52 J. D. Evans, C. J. Sumby and C. J. Doonan, Chem. Soc. Rev., 2014, 43, 5933-5951.

53 J. Juan-Alcaniz, J. Gascon, F. Kapteijn and J. Mater, Chem, 2012, 22, 10102-10118.

54 S. H. Jhung, N. A. Khan and Z. Hasan, CrystEngComm, 2012, 14, 7099-7109.

55 O. M. Yaghi, M. O’Keeffe, N. W. Ockwig, H. K. Chae, M. Eddaoudi and J. Kim, Reticular, Nature, 2003, 423, 705-714.

56 J. H. Cavka, S. Jakobsen, U. Olsbye, N. Guillou, C. Lamberti, S. Bordiga and K. P. Lillerud, J. Am. Chem. Soc., 2008, 130, 13850-13851.
57 P. D. C. Dietzel, P. A. Georgiev, J. Eckert, R. Blom, T. Strässle and T. Unruh, Chem. Commun., 2010, 46, 4962-4964.

58 D. Yu, A. O. Yazaydin, J. R. Lane, P. D. C. Dietzel and R. Q. Snurr, Chem. Sci., 2013, 4, 3544-3556.

59 S. M. Chavan, G. C. Shearer, E. Bloch and S. Bordiga, ChemPhysChem, 2012, 13, 445-448.

60 C. Volkringer, D. Popov, T. Loiseau, G. Férey, M. Burghammer, C. Riekel, M. Haouas and F. Taulelle, Chem. Mater., 2009, 21, 5695-5697.

61 G. Férey, C. Serre, C. M. Draznieks, F. Millange, S. Surblé, J. Dutour and I. Margiolaki, Angew. Chem. Int. Ed., 2004, 43, 6296-6301.

62 P. Horcajada, S. Surblé, C. Serre, D.-Y. Hong, Y.-K. Seo, J. S. Chang, J.-M. Grenèche, I. Margiolaki and G. Férey, Chem. Commun., 2007, 2820-2822.

63 T. Loiseau, L. Lecroq, C. Volkringer, J. Marrot, G. Férey, M. Haouas, F. Taulelle, S. Bourrelly, P. L. Llewellyn and M. Latroche, J. Am. Chem. Soc., 2006, 128, 10223-10230.

64 P. Long, H. Wu, Q. Zhao, Y. Wang, J. Dong and J. Li, Microporous Mesoporous Mater., 2011, 142, 489-493.

65 C. Volkringer, T. Loiseau, G. Férey, C. M. Morais, F. Taulelle, V. Montouillout and D. Massiot, Microporous Mesoporous Mater., 2007, 105, 111-117.

66 C. Volkringer and T. Loiseau, Mater. Res. Bull., 2006, 41, 948-954.

67 Z. Wang and S. M. Cohen, Chem. Soc. Rev., 2009, 38, 315-1329.

68 D.-Y. Du, J.-S. Qin, S.-L. Li, Z.-M. Su and Y.-Q. Lan, Chem. Soc. Rev., 2014, 43, 4615-4632.

69 M. G. Goestena, J. Juan-Alcaniz, E. V. Ramos-Fernandez, K. B. S. S. Gupta, E. Stavitski, H. V. Bekkum, J. Gascon and F. Kapteijn, J. Catal., 2011, 281, 177-187.

70 D. Britt, C. Lee, F. J. Uribe-Romo, H. Furukawa and O. M. Yaghi, Inorg. Chem., 2010, 49, 6387-6389.

71 M. Kandiah, S. Usseglio, S. Svelle, U. Olsbye, K. P. Lillerud and M. Tilset, J. Mater. Chem., 2010, 20, 9848-9851.

72 I. Ahmed, Z. Hasan, N. A. Khan and S. H. Jhung, Appl. Catal., B, 2013, 129, 123-129.

73 J. M. Taylor, R. Vaidhyanathan, S. S. Iremonger and G. K. H. Shimizu, J. Am. Chem. Soc., 2012, 134, 14338-14340.

74 J. Canivet, A. Fateeva, Y. Guo, B. Coasne and D. Farrusseng, Chem. Soc. Rev., 2014, 43, 5594-5617.

75 J. J. Low, A. I. Benin, P. Jakubczak, J. F. Abrahamian, S. A. Faheem and R. R. Willis, J. Am. Chem. Soc., 2009, 131, 15834-15842.

76 F. Jeremias, A. Khutia, S. K. Henninger and C. Janiak, J. Mater. Chem., 2012, 22, 10148-10151.

77 K. A. Cychosz and A. J. Matzger, Langmuir, 2010, 26, 17198-17202.

78 J. Ehrenmann, S. K. Henninger and C. Janiak, Eur. J. Inorg. Chem., 2011, 471-474.

79 K. S. Park, Z. Ni, A. P. Cote, J. Y. Choi, R. Huang, F. J. UribeRomo, H. K. Chae, M. O'Keeffe and O. M. Yaghi, Proc. Natl. Acad. Sci. U. S. A., 2006, 103, 10186-10191.

80 D.-D. Zu, L. Lu, X.-Q. Liu, D.-Y. Zhang and L.-B. Sun, J. Phys. Chem. C, 2014, 118, 19910-19917. 
81 T. Wu, L. Shen, M. Luebbers, C. Hu, Q. Chen, Z. Ni and R. I. Masel, Chem. Commun., 2010, 46, 6120-6122.

82 K. Sumida, D. L. Rogow, J. A. Mason, T. M. McDonald, E. D. Bloch, Z. R. Herm, T.-H. Bae and J. R. Long, Chem. Rev., 2012, 112, 724-778.

83 J.-R. Li, Y. Ma, M. C. McCarthy, J. Sculley, J. Yu, H.-K. Jeong, P. B. Balbuena and H.-C. Zhou, Coord. Chem. Rev., 2011, 255, 1791-1823.

84 N. A. Khan and S. H. Jhung, J. Hazard. Mater., 2013, 260, 1050-1056.

85 I. Ahmed, N. A. Khan and S. H. Jhung, Inorg. Chem., 2013, 52, 14155-14161.

86 C. Bao and C.-L. Fang, Water Resour. Manage. Ser., 2012, 26, 531-552.

87 X.-J. Zhang, C. Chen, P.-F. Lin, A.-X. Hou, Z.-B. Niu and J. Wang, Environ. Sci. Technol., 2011, 45, 161-167.

88 I. Michael, L. Rizzo, C. S. McArdell, C. M. Manaia, C. Merlin, T. Schwartz, C. Dagot and D. Fatta-Kassinos, Water Res., 2013, 47, 957-995.

89 Q. Bu, B. Wang, J. Huang, S. Deng and G. Yu, J. Hazard. Mater., 2013, 262, 189-211.

90 http://water.epa.gov/scitech/methods/cwa/pollutants.cfm.

91 L. Xie, D. Liu, H. Huang, Q. Yang and C. Zhong, Chem. Eng. J., 2014, 246, 142-149.

92 X. K. Zhao, G. P. Yang and X. C. Gao, Chemosphere, 2003, 52, 917-925.

93 J. Fawell and C. N. Ong, Int. J. Water Resour. D, 2012, 28, 247-263.

94 Y. M. Slokar and M. L. Marechal, Dyes Pigm., 1998, 37, 335-356.

95 M. Asgher and H. N. Bhatti, Waste biomass, Ecol. Eng., 2010, 36, 1660-1665.

96 D. Inthorn, S. Singhtho, P. Thiravetyan and E. Khan, Bioresour. Technol., 2004, 94, 299-306.

97 C. G. Daughton, Environ. Impact Assess., 2004, 24, 711-732.

98 C. G. Daughton, Renew. Resour. J., 2005, 23, 6-23.

99 E. M. Cuerda-Correaa, J. R. Domínguez-Vargas, F. J. OlivaresMarín and J. B. Heredia, J. Hazard. Mater., 2010, 177, 1046-1053.

100 O. A. H. Jones, N. Voulvoulis and J. N. Lester, Environ. Sci. Technol., 2005, 35, 401-427.

101 J. L. Packer, J. J. Werner, D. E. Latch, K. McNeill and W. A. Arnold, Aquat. Sci., 2003, 65, 342-351.

102 J. K. Fawell, D. Sheahan, H. A. James, M. Hurst and S. Scott, Water Res., 2001, 35, 1240-1244.

103 M. Carballa, F. Omil and J. M. Lema, Electron. J. Environ., Agric. Food Chem., 2003, 2, 309-313.

104 A. A. Al-Majed, A. R. Adebayo and M. E. Hossain, J. Environ. Manage., 2012, 113, 213-227.

105 A. Sarkar and S. Mahapatra, J. Mater. Chem. A, 2014, 2, 3808-3818.

106 J. W. Jun, M. Tong, B. K. Jung, Z. Hasan, C. Zhong and S. H. Jhung, Chem. - Eur. J., 2015, 21, 347-354.

107 M. Sarker, J. Y. Song and S. H. Jhung, J. Hazard. Mater., 2017, 335, 162-169.

108 B. K. Jung, J. W. Jun, Z. Hasan and S. H. Jhung, Chem. Eng. J., 2015, 267, 9-15.
109 C. Tian, J. Zhao, X. Ou, J. Wan, Y. Cai, Z. Lin, Z. Dang and B. Xing, Environ. Sci. Technol., 2018, 52, 3466-3475.

110 Y. Lv, R. Zhang, S. Zeng, K. Liu, S. Huang, Y. Liu, P. Xu, C. Lin, Y. Cheng and M. Liu, Chem. Eng. J., 2018, 339, 359-368.

111 I. Chaari, E. Fakhfakh, M. Medhioub and F. Jamoussi, J. Mol. Struct., 2019, 1179, 672-677.

112 M. A. Islam, M. J. Ahmed, W. A. Khanday, M. Asif and B. H. Hameed, J. Environ. Manage., 2017, 203, 237-244.

113 G. V. Brião, S. L. Jahn, E. L. Foletto and G. L. Dotto, Colloids Surf., A, 2018, 556, 43-50.

114 G. P. S. Aguiar, B. D. Arcari, L. M. Chaves, C. D. Magro, D. L. Boschetto, A. L. Piato and J. V. Oliveira, Ind. Crops Prod., 2018, 112, 1-5.

115 M. Ismail, Z. Wu, L. Zhang, J. Ma, Y. Jia, Y. Hu and Y. Wang, Chemosphere, 2019, 228, 212-218.

116 A. S. Moscofian, C. T. Pires, A. P. Vieira and C. Airoldi, RSC Adv., 2012, 2(8), 3502-3511.

117 N. Belhouchat, H. Zaghouane-Boudiaf and C. Viseras, Appl. Clay Sci., 2017, 135, 9-15.

118 Y. Li, A. R. Zimmerman, F. He, J. Chen, L. Han, H. Chen, X. Hu and B. Gao, Sci. Total Environ, 2020, 10, 137972.

119 C. Puri and G. Sumana, Appl. Clay Sci., 2018, 166, 102-112. 120 S. T. Akar and R. Uysal, Chem. Eng. J., 2010, 162, 591-598.

121 S. Bhasin, J. P. Brito, G. R. Cunningham, F. J. Hayes, H. N. Hodis, A. M. Matsumoto and M. A. Yialamas, J. Clin. Endocrinol. Metab., 2018, 103(5), 1715-1744.

122 A. S. Moscofian, C. T. Pires, A. P. Vieira and C. Airoldi, RSC Adv., 2012, 2, 3502-3511.

123 A. S. P. Moscofian, C. T. G. V. M. T. Pires, A. P. Vieira and C. Airoldi, RSC Adv., 2012, 2(8), 3502-3511.

124 W. Lu, Z. Wei, Z.-Y. Gu, T.-F. Liu, J. Park, J. Park, J. Tian, M. Zhang, Q. Zhang and T. Gentle III, Chem. Soc. Rev., 2014, 43, 5561-5593.

125 A. Mariyam, M. Shahid, I. Mantasha, M. S. Khan and M. Ahmad, J. Inorg. Organomet. Polym. Mater., 2020, 30, 1935-1943.

126 (a) Mantasha I., M. Shahid, H. A. M. Saleh, K. M. A. Qasem and M. Ahmad, CrystEngComm, 2020, 22, 3891-3909; (b) Mantasha I., H. A. M. Saleh, K. M. A. Qasem, M. Shahid and M. Ahmad, Inorg. Chim. Acta, 2020, 511, 119787.

127 K. Tan, N. Nijem, Y. Gao, S. Zuluaga, J. Li, T. Thonhauser and Y. J. Chabal, CrystEngComm, 2015, 17, 247-260.

128 X. Lan, H. Zhang, P. Bai and X. Guo, Microporous Mesoporous Mater., 2016, 231, 40-46.

129 P. M. Schoenecker, C. G. Carson, H. Jasuja, C. J. Flemming and K. S. Walton, Ind. Eng. Chem. Res., 2012, 51, 6513-6519.

130 D. Fröhlich, S. K. Henninger and C. Janiak, Dalton Trans., 2014, 43, 15300-15304.

131 L. Huang, H. Wang, J. Chen, Z. Wang, J. Sun, D. Zhao and Y. Yan, Microporous Mesoporous Mater., 2003, 58, 105-114.

132 J. A. Greathouse and M. D. Allendorf, J. Am. Chem. Soc., 2006, 128, 10678-10679.

133 J. B. DeCoste, G. W. Peterson, B. J. Schindler, K. L. Killops, M. A. Browe and J. J. Mahle, J. Mater. Chem. A, 2013, 1(1932), 11922-11932. 
134 K. A. Cychosz and A. J. Matzger, Langmuir, 2010, 26, 17198-17202.

135 G. Férey, C. Mellot-Draznieks, C. Serre, F. Millange, J. Dutour, S. Surblé and I. Margiolaki, Science, 2005, 309, 2040-2042.

136 J. H. Cavka, S. Jakobsen, U. Olsbye, N. Guillou, C. Lamberti, S. Bordiga and K. P. Lillerud, J. Am. Chem. Soc., 2008, 130, 13850-13851.

137 D. Feng, Z. Y. Gu, J. R. Li, H. L. Jiang, Z. Wei and H. C. Zhou, Angew. Chem., 2012, 124, 10453-10456.

138 D. Feng, W.-C. Chung, Z. Wei, Z.-Y. Gu, H.-L. Jiang, Y.-P. Chen, D. J. Darensbourg and H.-C. Zhou, J. Am. Chem. Soc., 2013, 135, 17105-17110.

139 C. Volkringer and S. M. Cohen, Angew. Chem. Int. Ed., 2010, 49, 4644-4648.

140 I. J. Kang, N. A. Khan, E. Haque and S. H. Jhung, Chem. Eur. J., 2011, 17, 6437-6442.

141 M. Kandiah, M. H. Nilsen, S. Usseglio, S. Jakobsen, U. Olsbye, M. Tilset, C. Larabi, E. A. Quadrelli, F. Bonino and K. P. Lillerud, Chem. Mater., 2010, 22, 6632-6640.

142 K. S. Park, Z. Ni, A. P. Côté, J. Y. Choi, R. Huang, F. J. UribeRomo, H. K. Chae, M. O'Keeffe and O. M. Yaghi, Proc. Natl. Acad. Sci. U. S. A., 2006, 103, 10186-10191.

143 D. Cunha, M. Ben Yahia, S. Hall, S. R. Miller, H. Chevreau, E. Elkaïm, G. Maurin, P. Horcajada and C. Serre, Chem. Mater., 2013, 25, 2767-2776.

144 S. Jung, Y. Kim, S.-J. Kim, T.-H. Kwon, S. Huh and S. Park, Chem. Commun., 2011, 47, 2904-2906.

145 S. S. Han, S.-H. Choi and A. C. van Duin, Chem. Commun., 2010, 46, 5713-5715.

146 M. De Toni, R. Jonchiere, P. Pullumbi, F. X. Coudert and A. H. Fuchs, ChemPhysChem, 2012, 13, 3497-3503.

147 J. Canivet, A. Fateeva, Y. Guo, B. Coasne and D. Farrusseng, Chem. Soc. Rev., 2014, 43, 5594-5617.

148 N. C. Burtch, H. Jasuja and K. S. Walton, Chem. Rev., 2014, 114, 10575-10612.

149 M. G. Goesten, F. Kapteijn and J. Gascon, CrystEngComm, 2013, 15, 9249-9257.

150 J. M. Taylor, R. Vaidhyanathan, S. S. Iremonger and G. K. Shimizu, J. Am. Chem. Soc., 2012, 134, 14338-14340.

151 J. Yang, A. Grzech, F. M. Mulder and T. J. Dingemans, Chem. Commun., 2011, 47, 5244-5246.

152 T. Wu, L. Shen, M. Luebbers, C. Hu, Q. Chen, Z. Ni and R. I. Masel, Chem. Commun., 2010, 46, 6120-6122.

153 H. Jasuja and K. S. Walton, J. Phys. Chem. C, 2013, 117, 7062-7068.

154 K. Wang, D. Feng, T.-F. Liu, J. Su, S. Yuan, Y.-P. Chen, M. Bosch, X. Zou and H.-C. Zhou, J. Am. Chem. Soc., 2014, 136, 13983-13986.

155 B. Wang, X.-L. Lv, D. Feng, L.-H. Xie, J. Zhang, M. Li, Y. Xie, J.-R. Li and H.-C. Zhou, J. Am. Chem. Soc., 2016, 138, 6204-6216.

156 J. H. Wang, M. Li and D. Li, Chem. - Eur. J., 2014, 20, 12004-12008.

157 S. Galli, N. Masciocchi, V. Colombo, A. Maspero, G. Palmisano, F. LopezGarzon, M. Domingo-García, I. Fernandez-Morales, E. Barea and J. Navarro, Chem. Mater., 2010, 22, 1664-1672.
158 V. Colombo, S. Galli, H. J. Choi, G. D. Han, A. Maspero, G. Palmisano, N. Masciocchi and J. R. Long, Chem. Sci., 2011, 2, 1311-1319.

159 H. Li, M. R. Hill, R. Huang, C. Doblin, S. Lim, A. J. Hill, R. Babarao and P. Falcaro, Chem. Commun., 2016, 52, 5973-5976.

160 H. Li, W. Shi, K. Zhao, H. Li, Y. Bing and P. Cheng, Inorg. Chem., 2012, 51, 9200-9207.

161 B. Chen, M. Eddaoudi, S. Hyde, M. O'Keeffe and O. Yaghi, Science, 2001, 291, 1021-1023.

162 D. M. Driscoll, D. Troya, P. M. Usov, A. J. Maynes, A. J. Morris and J. R. Morris, J. Phys. Chem. C, 2018, 122, 14582-14589.

163 W. Liang, C. J. Coghlan, F. Ragon, M. Rubio-Martinez, D. M. D'Alessandro and R. Babarao, Dalton Trans., 2016, 45, 4496-4500.

164 J. Qiu, Y. Feng, X. Zhang, M. Jia and J. Yao, J. Colloid Interface Sci., 2017, 499, 151-158.

165 L. Zhou, X. Zhang and Y. Chen, Mater. Lett., 2017, 197, 167-170. 166 H. Wu, Y. S. Chua, V. Krungleviciute, M. Tyagi, P. Chen, T. Yildirim and W. Zhou, J. Am. Chem. Soc., 2013, 135, 10525-10532.

167 S. Hu, M. Liu, X. Guo, K. Li, Y. Han, C. Song and G. Zhang, Cryst. Growth Des., 2017, 17, 6586-6595.

168 J. Ren, N. M. Musyoka, H. W. Langmi, T. Segakweng, B. C. North, M. Mathe and X. Kang, Int. J. Hydrogen Energy, 2014, 39, 12018-12023.

169 K. Wang, C. Li, Y. Liang, T. Han, H. Huang, Q. Yang, D. Liu and C. Zhong, Chem. Eng. J., 2016, 289, 486-493.

170 F. Vermoortele, B. Bueken, G. L. Bars, B. Van de Voorde, M. Vandichel, K. Houthoofd, A. Vimont, M. Daturi, M. Waroquier, V. Van Speybroeck, C. Kirschhock and D. E. De Vos, J. Am. Chem. Soc., 2013, 135, 11465-11468.

171 P. Ghosh, Chem. Commun., 2014, 50, 11329-11331.

172 D. Jiang, M. Chen, H. Wang, G. Zeng, D. Huang, M. Cheng, Y. Liu, W. Xue and Z. W. Wang, Coord. Chem. Rev., 2019, 380, 471-483.

173 Y. Li, Y. Liu, W. Gao, L. Zhang, W. Liu, J. Lu, Z. Wang and Y.-J. Deng, CrystEngComm, 2014, 16, 7037-7042.

174 Z. Xie, W. Xu, X. Cui and Y. Wang, ChemSusChem, 2017, 10, 1645-1663.

175 W. Teng, N. Bai, Z. Chen, J. Shi, J. Fan and W.-X. Zhang, Chem. Eng. J., 2018, 346, 388-396.

176 B. N. Bhadra and S. H. Jhung, Microporous Mesoporous Mater., 2018, 270, 102-108.

177 J. Y. Song, B. N. Bhadra, N. A. Khan and S. H. Jhung, Microporous Mesoporous Mater., 2018, 260, 1-8.

178 A. Almasoudi and R. Mokaya, J. Mater. Chem., 2012, 22, 146-152.

179 R. R. Salunkhe, Y. V. Kaneti, J. Kim, J. H. Kim and Y. Yamauchi, Acc. Chem. Res., 2016, 49, 2796-2806.

180 Z. Abbasi, E. Shamsaei, S. K. Leong, B. Ladewig, X. Zhang and H. Wang, Microporous Mesoporous Mater., 2016, 236, 28-37.

181 H. Y. Lin, J. Zhao, G. Song, J. Luan, X. X. Liu and G. C. Liu, Dalton Trans., 2017, 46, 17067-17073. 
182 S. Xu, Y. Lv, X. Zeng and D. Cao, Chem. Eng. J., 2017, 323, 502-511.

183 Z. Song, W. Liu, N. Cheng, M. N. Banis, X. Li, Q. Sun, B. Xiao, Y. Liu, A. Lushington, R. Li, L. Liu and X. Sun, Mater. Horiz., 2017, 4, 900-907.

184 N. L. Torad, M. Hu, S. Ishihara, H. Sukegawa, A. A. Belik, M. Imura, K. Ariga, Y. Sakka and Y. Yamauchi, Small, 2014, 10, 2096-2107.

185 L. Jin, X. Zhao, X. Qian and M. Dong, J. Colloid Interface Sci., 2018, 509, 245-253.

186 J. Zhang, X. Yan, X. Hu, R. Feng and M. Zhou, Chem. Eng. J., 2018, 347, 640-647.

187 Y. Song, T. Qiang, M. Ye, Q. Ma and Z. Fang, Appl. Surf. Sci., 2015, 359, 834-840.

188 E. Abdelillah Ali Elhussein, S. Sahin and S. S. Bayazit, J. Mol. Liq., 2018, 255, 10-17.

189 S. Ru, L. Xu, H. Xiao and X. Li, Mater. Res. Express, 2016, 3, 115006.

190 Y. Gu, K. Cheng, Y.-N. Wu, Y. Wang, C. Morlay, F. Li and A. C. S. Sust, Chem. Eng., 2016, 4, 6744-6753.

191 E. Yllmaz, E. Sert and F. S. Atalay, J. Taiwan Inst. Chem. Eng., 2016, 65, 323-330.

192 E. Haque, J. W. Jun and S. H. Jhung, J. Hazard. Mater., 2011, 185, 507-511.

193 M. Tong, D. Liu, Q. Yang, S. Devautour-Vinot, G. Maurin and C. Zhong, J. Mater. Chem. A, 2013, 1, 8534.

194 S. Lin, Z. Song, G. Che, A. Ren, P. Li, C. Liu and J. Zhang, Microporous Mesoporous Mater., 2014, 193, 27-34.

195 S. Duan, J. Li, X. Liu, Y. Wang, S. Zeng, D. Shao and T. Hayat, ACS Sustainable Chem. Eng., 2016, 4, 3368-3378.

196 A. C. Tella, M. D. Olawale, M. Neuburger and J. A. Obaleye, J. Solid State Chem., 2017, 255, 157-166.

197 D. M. Driscoll, D. Troya, P. M. Usov, A. J. Maynes, A. J. Morris and J. R. Morris, J. Phys. Chem. C, 2018, 122, 14582-14589.

198 W. Liang, C. J. Coghlan, F. Ragon, M. Rubio-Martinez, D. M. D'Alessandro and R. Babarao, Dalton Trans., 2016, 45, 4496-4500.

199 K.-W. Jung, B. H. Choi, C. M. Dao, Y. J. Lee, J.-W. Choi, K.-H. Ahn and S.-H. Lee, J. Ind. Eng. Chem., 2018, 59, 149-159.

200 Y. Zhou, S. Yao, Y. Ma, G. Li, Q. Huo and Y. Liu, Chem. Commun., 2018, 54, 3006-3009.

201 J. He, J. Li, W. Du, Q. Han, Z. Wang and M. Li, J. Alloys Compd., 2018, 750, 360-367.

202 Y. H. Tang, F. Wang and J. Zhang, Dalton Trans., 2018, 47. 203 Y. Deng, Y. Zhao, P. Wang, Z.-Y. Yao, X.-D. Zhang and W.-Y. Sun, Microporous Mesoporous Mater., 2017, 241, 192-201.

204 L. Yang, X. Li, C.-Y. Sun, H. Wu, C.-G. Wang and Z.-M. Su, New J. Chem., 2017, 41, 3661-3666.

205 M. Guo, S. Liu, H. Guo, Y. Sun, X. Guo and R. Deng, Dalton Trans., 2017, 46, 14988-14994.

206 G. G. Sezer, M. Arıcı, I. Erucar, O. Z. Yesilel, H. U. Özel, B. T. Gemici and H. Erer, J. Solid State Chem., 2017, 255, 89-96.

207 S. Phengthaisong, A. Cheansirisomboon, J. Boonmak and S. Youngme, Inorg. Chim. Acta, 2018, 479, 172-178.
208 S. Chand, S. M. Elahi, A. Pal and M. C. Das, Dalton Trans., 2017, 46, 9901-9911.

209 N. Ahmad, H. A. Younus, A. H. Chughtai, K. V. Hecke, Z. A. K. Khattak, Z. Gaoke, M. Danish and F. Verpoort, Catal. Sci. Technol., 2018, 8, 4010-4017.

210 M.-J. Tsai and J.-Y. Wu, Polymers, 2017, 9, 661.

211 Y. Rachuri, S. Subhagan, B. Parmar, K. K. Bisht and E. Suresh, Dalton Trans., 2018, 47, 898-908.

212 E. Haque, V. Lo, A. I. Minett, A. T. Harris and T. L. Church, J. Mater. Chem. A, 2014, 2, 193-203.

213 M. Roushani, Z. Saedi and T. M. Beygi, J. Taiwan Inst. Chem. Eng., 2016, 66, 164-171.

214 Q. Chen, Q. He, M. Lv, Y. Xu, H. Yang, X. Liu and F. Wei, Appl. Surf. Sci., 2015, 327, 77-85.

215 R. Bibi, L. Wei, Q. Shen, W. Tian, O. Oderinde, N. Li and J. Zhou, J. Chem. Eng. Data, 2017, 62, 1615-1622.

216 M. Oveisi, M. A. Asli and N. M. Mahmoodi, J. Hazard. Mater., 2018, 347, 123-140.

217 D. Jiang, M. Chen, H. Wang, G. Zeng, D. Huang, M. Cheng, Y. Liu, W. Xue and Z. Wang, Coord. Chem. Rev., 2019, 380, 471-483.

218 J. Hu, H. Yu, W. Dai, X. Yan, X. Hu and H. Huang, RSC Adv., 2014, 4, 35124-35130.

219 H. M. Abd El Salam, S. A. Younis, H. R. Ali and T. Zaki, Microporous Mesoporous Mater., 2017, 241, 210-217.

220 Y. Han, M. Liu, K. Li, Q. Sun, W. Zhang, C. Song, G. Zhang, Z. C. Zhang and X. Guo, Inorg. Chem. Front., 2017, 4, 1870-1880.

221 J. M. Yang, R. J. Ying, C. X. Han, Q. T. Hu, H. M. Xu, J. H. Li, Q. Wang and W. Zhang, Dalton Trans., 2018, 47, 3913-3920.

222 J. Y. Song, I. Ahmed, P. W. Seo and S. H. Jhung, ACS Appl. Mater. Interfaces, 2016, 8, 27394-27402.

223 X. Zhao, K. Wang, Z. Gao, H. Gao, Z. Xie, X. Du and H. Huang, Ind. Eng. Chem. Res., 2017, 56, 4496-4501.

224 X.-P. Luo, S.-Y. Fu, Y.-M. Du, J.-Z. Guo and B. Li, Microporous Mesoporous Mater., 2017, 237, 268-274.

225 J. Qiu, Y. Feng, X. Zhang, M. Jia and J. Yao, J. Colloid Interface Sci., 2017, 499, 151-158.

226 L. Zhou, X. Zhang and Y. Chen, Mater. Lett., 2017, 197, 167-170.

227 J. Ren, N. M. Musyoka, H. W. Langmi, T. Segakweng, B. C. North, M. Mathe and X. Kang, Int. J. Hydrogen Energy, 2014, 39, 12018-12023.

228 K. Wang, C. Li, Y. Liang, T. Han, H. Huang, Q. Yang, D. Liu and C. Zhong, Chem. Eng. J., 2016, 289, 486-493.

229 P. Ghosh, Chem. Commun., 2014, 50, 11329-11331.

230 Z. Hasan and S. H. Jhung, J. Hazard. Mater., 2015, 283, 329-339.

231 E. Haque, J. E. Lee, I. T. Jang, Y. K. Hwang, J.-S. Chang, J. Jegal and S. H. Jhung, J. Hazard. Mater., 2010, 181, 535-542.

232 A. A. Adeyemo, I. O. Adeoye and O. S. Bello, Toxicol. Environ. Chem., 2012, 94, 1846-1863.

233 E. Haque, V. Lo, A. I. Minett, A. T. Harris and T. L. Church, J. Mater. Chem. A, 2014, 2, 193-203. 
234 E. Haque, J. W. Jun and S. H. Jhung, J. Hazard. Mater., 2011, 185, 507-511.

235 S. Lin, Z. Song, G. Che, A. Ren, P. Li, C. Liu and J. Zhang, Microporous Mesoporous Mater., 2014, 193, 27-34.

236 K. Iman, M. Shahid, M. S. Khan, M. Ahmad and F. Sama, CrystEngComm, 2019, 21, 5299-5309.

237 Z. Hasan, E.-J. Choi and S. H. Jhung, Chem. Eng. J., 2013, 219, 537-544.

238 Z. Hasan, J. Jeon and S. H. Jhung, J. Hazard. Mater., 2012, 209-210, 151-157.

239 I. Ahmed, Z. Hasan, N. A. Khan and S. H. Jhung, Appl. Catal., B, 2013, 129, 123-129.

240 B. Liu, F. Yang, Y. Zou and Y. Peng, J. Chem. Eng. Data, 2014, 59, 1476-1482.

241 L. Xie, D. Liu, H. Huang, Q. Yang and C. Zhong, Chem. Eng. J., 2014, 246, 142-149.

242 C. Petit and T. J. Bandosz, Adv. Funct. Mater., 2010, 20, 111-118.

243 N. Planas, A. L. Dzubak, R. Poloni, L.-C. Lin, A. McManus, T. M. McDonald, J. B. Neaton, J. R. Long, B. Smit and L. Gagliardi, J. Am. Chem. Soc., 2013, 135, 7402-7405.

244 Mantasha I., S. Hussain, M. Ahmad and M. Shahid, Sep. Purif. Technol., 2020, 238, 116413.

245 N. A. Khan and S. H. Jhung, Angew. Chem. Int. Ed., 2012, 51, 1198-1201.

246 I. Ahmed and S. H. Jhung, Chem. Eng. J., 2014, 251, 35-42. 247 F.-X. Qin, S.-Y. Jia, Y. Liu, H.-Y. Li and S.-H. Wu, Desalin. Water Treat., 2014, 54, 93-102.

248 E. Y. Park, Z. Hasan, N. A. Khan and S. H. Jhung, J. Nanosci. Nanotechnol., 2013, 13, 2789-2794.

249 S.-H. Huo and X.-P. Yan, J. Mater. Chem., 2012, 22, 7449-7455.

250 M. S. Khan, M. Khalid, M. S. Ahmad, M. Shahid and M. Ahmad, Dalton Trans., 2019, 48, 12918-12932.

251 C. Yang, U. Kaipa, Q. Z. Mather, X. Wang, V. Nesterov, A. F. Venero and M. A. Omary, J. Am. Chem. Soc., 2011, 133, 18094-18097.

252 K.-Y. A. Lin, H. Yang, C. Petit and F.-K. Hsu, Chem. Eng. J., 2014, 249, 293-301.

253 U. I. Gaya and A. H. Abdullah, J. Photochem. Photobiol., C, 2008, 9, 1-12.
254 H. Yang and H. Cheng, Sep. Purif. Technol., 2007, 56, 392-396.

255 M. C. Das, H. Xu, Z. Wang, G. Srinivas, W. Zhou, Y.-F. Yue, V. N. Nesterov, G. Qian and B. Chen, Chem. Commun., 2011, 47, 11715-11717.

256 G. Li, M. Yu, Z. Wang, J. Lin, R. Wang and J. Fang, J. Nanosci. Nanotechnol., 2006, 6, 1416-1422.

257 J. Lu, J.-X. Lin, X.-L. Zhao and R. Cao, Chem. Commun., 2012, 48, 669-671.

258 M. R. Hoffmann, S. T. Martin, W. Choi and D. W. Bahnemann, Chem. Rev., 1995, 95, 69-96.

259 T. L. Thompson and J. T. Yates, Chem. Rev., 2006, 106, 4428-4453.

260 A. Mills and S. Le Hunte, J. Photochem. Photobiol., A, 1997, 108, 1-35.

261 K. Ayoub, E. D. van Hullebusch, M. Cassir and A. Bermond, J. Hazard. Mater., 2010, 178, 10-28.

262 U. Akpan and B. Hameed, J. Hazard. Mater., 2009, 170, 520-529.

263 A. Janczyk, E. Krakowska, G. Stochel and W. Macyk, J. Am. Chem. Soc., 2006, 128, 15574-15575.

264 A. Y. Nosaka, E. Kojima, T. Fujiwara, H. Yagi, H. Akutsu and Y. Nosaka, J. Phys. Chem. B, 2003, 107, 12042-12044.

265 M. Alvaro, E. Carbonell, B. Ferrer, F. X. Llabrés i Xamena and H. Garcia, Chem. - Eur. J., 2007, 13, 5106-5112.

266 M. C. Das, H. Xu, Z. Wang, G. Srinivas, W. Zhou, Y.-F. Yue, V. N. Nesterov, G. Qian and B. Chen, Chem. Commun., 2011, 47, 11715-11717.

267 J. M. Joseph, H. Destaillats, H.-M. Hung and M. R. Hoffmann, J. Phys. Chem. A, 2000, 104, 301-307.

268 L. S. Cui, X. M. Meng, Y. G. Li, K. R. Huang, Y. C. Li, J. Q. Long and P. F. Yao, CrystEngComm, 2019, 21, 3798-3809.

269 H. Kaur, R. Kumar, A. Kumar, V. Krishnan and R. R. Koner, Dalton Trans., 2019, 48, 915-927.

270 T. Zeng, L. Wang, L. Feng, H. Xu, Q. Cheng and Z. Pan, Dalton Trans., 2019, 48, 523-534.

271 T. L. Easun, F. Moreau, Y. Yan, S. Yang and M. Schröder, Chem. Soc. Rev., 2017, 46, 239-274.

272 (a) J.-C. Jin, J. Wu, Y.-X. He, B.-H. Li, J.-Q. Liu, R. Prasad, A. Kumar and S. R. Batten, CrystEngComm, 2017, 19, 6464-6472; (b) Y. Pan, Q. Ding, H. Xu, C. Shi, A. Singh, A. Kumar and J. Liu, CrystEngComm, 2019, 21, 4578-4585. 